\title{
A Computational Approach to Modeling Cellular- Scale Blood Flow in Complex Geometry
}

\author{
Peter Balogh and Prosenjit Bagchi ${ }^{1}$ \\ Mechanical and Aerospace Engineering Department \\ Rutgers, The State University of New Jersey \\ Piscataway, NJ 08854, USA
}

${ }^{1}$ Corresponding author. Email: pbagchi@jove.rutgers.edu

(C) 2017. This manuscript version is made available under the Elsevier user license http://www.elsevier.com/open-access/userlicense/1.0/ 
ABSTRACT: We present a computational methodology for modeling cellular-scale blood flow in arbitrary and highly complex geometry. Our approach is based on immersedboundary methods, which allow modeling flows in arbitrary geometry while resolving the large deformation and dynamics of every blood cell with high fidelity. The present methodology seamlessly integrates different modeling components dealing with stationary rigid boundaries of complex shape, moving rigid bodies, and highly deformable interfaces governed by nonlinear elasticity. Thus it enables us to simulate 'whole' blood suspensions flowing through physiologically realistic microvascular networks that are characterized by multiple bifurcating and merging vessels, as well as geometrically complex lab-on-chip devices. R2C1 The focus of the present work is on the development of a versatile numerical technique that is able to consider deformable cells and rigid bodies flowing in three-dimensional arbitrarily complex geometries over a diverse range of scenarios. After describing the methodology, a series of validation studies are presented against analytical theory, experimental data, and previous numerical results. Then, the capability of the methodology is demonstrated by simulating flows of deformable blood cells and heterogeneous cell suspensions in both physiologically realistic microvascular networks and geometrically intricate microfluidic devices. It is shown that the methodology can predict several complex microhemodynamic phenomena observed in vascular networks and microfluidic devices. The present methodology is robust and versatile, and has the potential to scale up to very large microvascular networks at organ levels. 


\section{Introduction}

Computational modeling of cellular-scale blood flow continues to pose new challenges despite significant progress made in recent years $[1,2]$. Blood at the cellular-scale behaves as a concentrated suspension that is primarily comprised of red blood cells (RBC), white blood cells (WBC), and platelets. The size of these cells is comparable to a typical vessel radius that is encountered in microcirculation and microfluidic devices. Red blood cells, the major constituent of blood, are extremely deformable particles, and this property is critical to many physiological functions [3-7]. Extreme deformation allows them to squeeze through narrow capillaries in microcirculation without getting damaged. It also causes platelets and WBCs to marginate towards the wall of a blood vessel. The margination of platelets is the first step in the formation of a blood clot, and the margination of WBCs is the first step in the body's defense against bacteria. High fidelity computational modeling of RBC deformation in three-dimensions (3D) and its extension to multiple cells in concentrated suspensions remains a non-trivial task. Additionally, the physical properties, e.g. size, shape, and deformability, of RBCs, WBCs, and platelets are widely different. As a result, 'whole' blood behaves as a poly-disperse suspension leading to further difficulty in modeling.

Another major computational challenge in modeling blood flow in microcirculation arises from the geometric complexity and network-like structure of blood vessels. Blood vessels in microcirculation are not simply straight tubes; rather, they continually bifurcate and

merge with other vessels resulting in a complex network [3-6]. This network structure is referred to as the microvasculature, and it varies from organ to organ. The architectural complexity increases significantly in tumor microvasculature, where vascular trifurcations and short-length shunts may be present. The influence of the network architecture on the flow of blood cells is expected to be significant, although it is not well understood. 
Geometric complexity is also present in microfluidic lab-on-chip devices handling blood. Examples include microchannels with sequential T-bifurcations for cell-plasma separation, channels with repeated expansions and contractions for inertial focusing of cells, and spiraling microchannels for isolating tumor cells [8]. Other examples include artificial vascular networks that are fabricated in an attempt to mimic the real microvasculature $[9,10]$.

To date, most modeling studies on the flow of deformable blood cells have been limited to flow in simple geometries, such as single, unbranched, straight vessels of uniform circular cross-section, and parallel-plate geometry [1,2]. Studies of deformable cells in complex geometry are scarce, and are usually limited to a relatively modest complexity, such as a single bifurcation. The motion of an isolated deformable capsule in 2D bifurcating channels was studied using a finite element model [11], boundary integral simulations [12], and lattice-Boltzmann immersed boundary simulations [13], and in 3D using dissipative particle dynamics [14]. Relatively simpler 2D vascular networks having two to three bifurcations were also considered using lattice-Boltzmann methods [15]. Axisymmetric motion of a single deformable capsule in a hyperbolic constriction was studied using the boundary integral method [16], while 3D capsule motion in constricted microchannels was studied using spectral boundary integral methods $[17,18]$. The boundary integral method was also used to study the deformation of an RBC squeezing through a narrow passage [19], the motion of a deformable capsule through a square duct with a corner [20], and the interaction of deformable RBCs with platelets flowing in a stenosed capillary vessel [21]. Dissipative particle dynamics has been used to simulate the motion of rigid and deformable particles and cells flowing through cylindrical microchannels, and arrays of posts with different geometrical cross-sections $[22,23]$. The lattice-Boltzmann immersed-boundary method has been utilized to study the 2D flow of multiple deformable RBCs in stenosed microvessels [24], and the interaction of RBCs and platelets near an intravascular thrombus [25].

Evidently, computational modeling of cellular-scale blood flow in 3D arbitrarily com- 
plex geometries, such as physiologically realistic microvascular networks characterized by multiple bifurcations and mergers, or highly complex microfluidic devices, is lacking. To bridge this gap, we present here a 3D direct numerical simulation approach based on the immersed boundary method (IBM) for simulating cellular-scale blood flow in complex geometries. The major strength of immersed boundary methods is that they permit simulation of flows involving arbitrarily complex boundaries without requiring a body-fitted computational mesh $[26,27]$. A common means of classifying such methods is to consider continuous forcing methods and direct forcing methods. While the continuous forcing methods are well suited for flows with elastic boundaries, such as highly deformable cell membranes [28-31], they are not well suited for simulating rigid boundaries. Direct forcing methods, however, are very well suited for simulating rigid boundaries [26]. In the present problem we consider blood as a suspension of red blood cells (RBC), white blood cells (WBC), and platelets. While the RBCs are extremely deformable, WBCs are relatively less deformable, and inactivated platelets are nearly rigid. Thus, from the immersed-boundary perspective, the problem involves three types of interfaces: deformable interfaces of the RBCs and WBCs that are governed by nonlinear elasticity, moving rigid boundaries of the platelets, and non-moving but geometrically complex boundaries such as vascular network walls. The numerical methodology for the present work builds on the immersed boundary methodologies of both the continuous and direct forcing types, and seamlessly integrates all of these modeling components. Using this approach enables us to simulate geometries that are arbitrarily complex, and not limited to boundaries defined analytically. This permits the simulation of actual physiological geometry, without requiring any simplifications or assumptions, while simultaneously resolving the motion and large deformation of each individual blood cell with high fidelity.

The scientific objective of the present work is to develop a novel numerical technique for simulating the flow of deformable cells and rigid bodies in arbitrarily complex geometries as encountered in diverse types of scenarios ranging from microvascular networks to 
microfluidic devices. This objective requires that we demonstrate the versatility of the methodology in dealing with diverse types of interfaces and geometries. The numerical methodology is presented in the next section, followed by a detailed validation in $\S I I I$ against analytical solutions, experimental results, and computational results. Then, in $\S I V$ we demonstrate the capabilities of the methodology using several examples, ranging from the flow of deformable RBCs in a physiologically similar microvascular network, to cell motion in a complex microfluidic device. Lastly, in Section V we discuss the various features that have been demonstrated with the current technique, and compare our approach to other commonly used methods.

\section{Numerical methodology}

\section{A. Overview}

The generic problem of cellular-scale blood flow in complex geometry is schematically shown in figure 1. We consider blood as a suspension of red blood cells (RBC), white blood cells (WBC), and platelets, with the suspending fluid being the plasma. As illustrated in figure 1, the problem involves three types of boundaries: (i) deforming cellular membranes, (ii) rigid but moving boundaries, and (iii) rigid, non-moving but geometrically complex boundaries such as vascular walls. The deforming interface is primarily the membranes of the red blood cells, which are extremely deformable. Activated white blood cells, and other circulating elements, such as vesicles, are also deformable. The membrane deformation of these cells is governed by complex nonlinear (visco)-elasticity. Inactivated platelets, in contrast, behave like rigid particles. The vascular walls are considered to be rigid for the present study. We use the continuous-forcing and direct-forcing immersed-boundary methods to integrate these diverse types of interfaces. Structurally, the RBCs are viscous liquid drops surrounded 


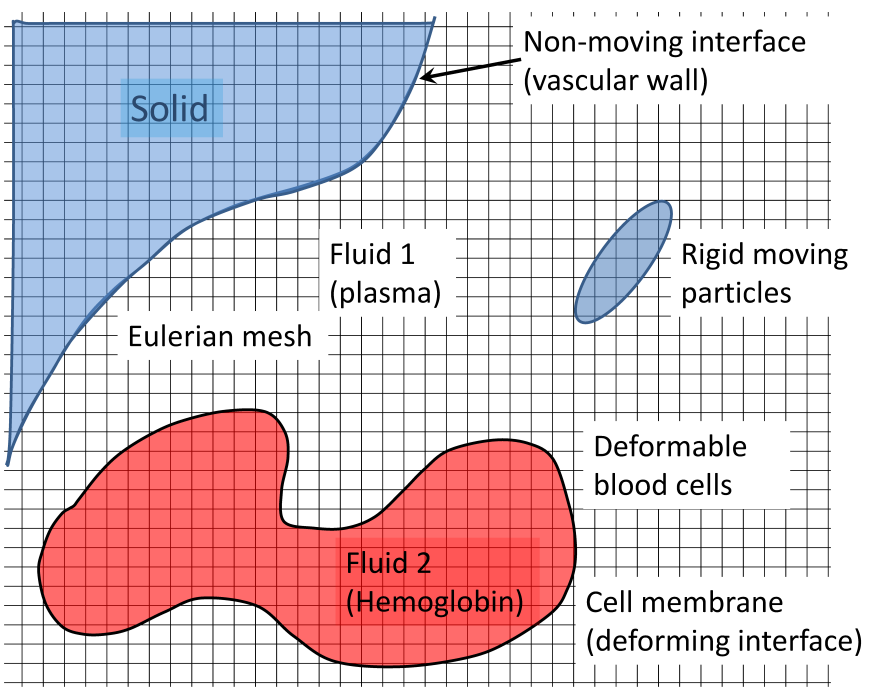

Figure 1: Schematic describing the generic problem of cellular-scale blood flow in complex geometry. The problem involves deformable interfaces (membranes of red blood cells and other deformable cells), moving rigid bodies (e.g., inactivated platelets), and non-moving vascular walls. The problem also involves multiple fluids of different viscosities. Boundaries are immersed in a fixed (Eulerian) rectangular Cartesian mesh that is used to discretize the entire computational domain.

by hyperelastic membranes. The liquid interior of the cell is the hemoglobin which has a different viscosity than the outside fluid plasma. Viscosity of the cytoplasmic fluids of the WBC and other circulating cells also differs from that of the plasma. Thus the problem involves multiple fluids of different viscosity.

The different types of interfaces present in the problem require different numerical treatments. A finite element method (FEM) is used to obtain the stresses generated in the deforming cell membranes. The membrane stresses are then coupled to the fluid flow by the continuous-forcing IBM. A sharp-interface ghost-node immersed-boundary method (GNIBM) is used to treat the non-moving vascular walls and the moving rigid objects. The flow solver is based on a coupled finite-volume/spectral method. In the following sections, each of these modeling components is described. Section B first discusses the new developments made in implementing these modeling components, in addition to specific 
considerations with regard to the fundamental approach. The methodology associated with deformable cells has been developed previously[30,36,37,49], and is discussed in Section C. Section D details a methodology for extending this approach into a computational framework that can also model complex rigid interfaces, both stationary and moving.

\section{B. Developments and considerations}

General numerical developments and considerations are now discussed with regard to implementing each of the modeling components.

For complex rigid interfaces, the general idea is adapted from that developed in [41], whereby a constraint is enforced at certain Eulerian grid points such that desired boundary conditions are achieved at the interfaces. In adapting this methodology, we have developed an explicit method of enforcing the constraints associated with the rigid interfaces. This does not require modifying the linear system on account of the rigid boundaries and thus permits use of efficient inversion techniques for solving the system. With regard to moving rigid objects, a novel methodology is considered to couple the rigid body dynamics using the Euler parameters with the fluid flow. This incurs minimal additional computational overhead and can in principle be applied to rigid bodies of any shape, not just the ones considered here. The types of complex stationary geometries considered here represent the first of their kind to be modeled with this type of method, namely complex microvascular networks. The procedure detailed later provides an efficient approach to incorporating these physiological structures into a simulation.

The implementation of the deformable cell methodology has been done in prior works $[30,36,37,49]$ in which a co-located arrangement of the velocity and pressure are used for 
the Eulerian grid on which the governing equations are solved. When using a co-located arrangement of variables one runs the risk of encountering the classic oscillations in the pressure field [61] on account of the discrete form of the Laplacian required to satisfy the continuity equation to machine epsilon. This issue is not encountered if only deformable interfaces are present due to the diffuse nature of continuous-forcing type methods. With the introduction of complex rigid interfaces to the problem, however, oscillations in the pressure field were found to result when tested. A pressure-velocity coupling algorithm was implemented to mitigate this, which resulted in a smooth pressure field for all simulations tested. However, the de-coupling between the pressure and velocity fields resulted in the rigid interface constraints being only weakly enforced using our explicit technique, when tested on highly complex geometries. This is primarily due to the fact that the discrete velocity field that is solenoidal is different from the velocity field associated with the momentum forcing, where the rigid interface constraints are enforced.

We have found that use of a staggered-grid arrangement of variables provides a highly robust means of simulating the complex rigid interfaces using our explicit technique for enforcing the rigid interface constraints. A well known benefit of a staggered arrangement is that it obviates the need for any special pressure-velocity coupling on account of the discrete form of the Laplacian being naturally compact. As such, there is an inherently strong coupling between the velocity and pressure fields. This permits simulations involving highly complex rigid geometries using our explicit technique to enforce the constraints. It is also highly desirable with regard to the methodology for moving rigid particles where the pressure is directly used to compute the motion of the particle, as discussed later. 


\section{Modeling Deformable Blood Cells}

\section{C.1. Principles of Approach}

$\mathrm{R} 1 \mathrm{C} 2$

Deformable blood cells are modeled as capsules, i.e., viscous drops enclosed by zerothickness hyperelastic membranes. In this model, the ultra-fine structure of the cell membrane, namely the lipid bilayers and the spectrin network, are indistinguishable. Below we limit our discussion to the modeling of an RBC; a similar approach is taken for modeling other deformable cells. The resting shape of the RBC is taken as the experimentally observed biconcave discocyte with an end-to-end distance of $7.8 \mu \mathrm{m}$, surface area $134.1 \mu \mathrm{m}^{2}$, and volume $94.1 \mu \mathrm{m}^{3}$ (figure 2a) [5]. The fluids both interior (hemoglobin) and exterior (plasma) to the cells are assumed to be incompressible and Newtonian. The RBC membrane is assumed to resist shear deformation, area dilatation, and bending. The resistance against shear deformation and area dilatation can be modeled using a suitable constitutive relation for the strain energy function $W_{s}$ of the membrane material. A common form of $W_{s}$ that is often used is based on the work of Skalak et al. [32] as

$$
W_{s}=\frac{G_{s}}{4}\left[\left(I_{1}^{2}+2 I_{1}-2 I_{2}\right)+C I_{2}^{2}\right]
$$

Here $G_{s} \sim 2-5 \times 10^{-6} \mathrm{~N} / \mathrm{m}$ is the membrane shear elastic modulus, $I_{1}=\epsilon_{1}^{2}+\epsilon_{2}^{2}-2$ and $I_{2}=\epsilon_{1}^{2} \epsilon_{2}^{2}-1$ are the strain invariants of the Green strain tensor, and $\epsilon_{1}$ and $\epsilon_{2}$ are the principal stretch ratios. The Green strain tension is defined as $\mathbf{E}=\left(\mathbf{F}^{\mathrm{T}} \cdot \mathbf{F}-\mathbf{I}\right)$, where $\mathbf{F}=\partial \mathbf{x} / \partial \mathbf{X}$ is the deformation gradient of the current configuration $\mathbf{x}$ relative to the original configuration $\mathbf{X}$ of the membrane (figure 2). The RBC membrane can undergo large shear deformation but almost negligible surface area dilation. In Eq. 1, the parameter $C$ is used to control the amount of surface area dilation, and a large value of $C$ results in a small area dilation. Other forms of $W_{s}$ have also been tested for the RBC membrane, and can be used within the framework of the present methodology. For example, a higher-order 
(a)

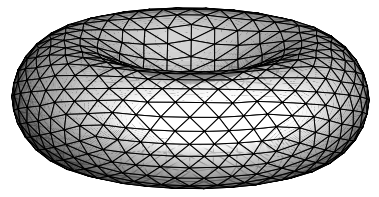

(b)

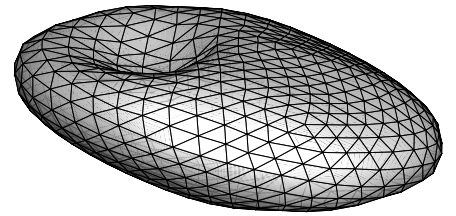

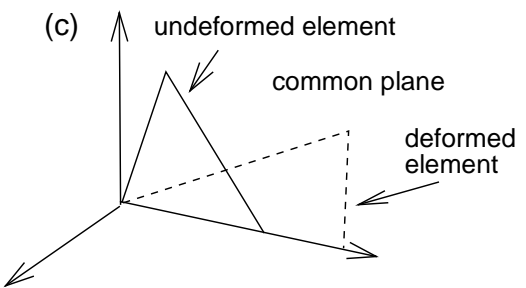

Figure 2: (a) Undeformed shape and Lagrangian mesh on the surface of a red blood cell. (b) An arbitrary deformed cell. (c) Deformed and undeformed Delauney triangles.

neo-Hookean model can be expressed as

$$
W_{s}=\frac{G_{s}}{2}\left(\epsilon_{1}^{2}+\epsilon_{2}^{2}+\frac{1}{\epsilon_{1}^{2} \epsilon_{2}^{2}}-3\right)+C_{3}\left(\epsilon_{1}^{2}+\epsilon_{2}^{2}+\frac{1}{\epsilon_{1}^{2} \epsilon_{2}^{2}}-3\right)^{3}
$$

where $C_{3}$ is a constant that was experimentally determined as $\sim G_{s} / 30$ [33]. The experi- R2C6 mental study in [33] has shown that at extreme deformation, the generalized neo-Hookean model works well for RBC deformation. In the present manuscript, we have used this law for the micropipette aspiration simulations (§III.C), since the RBC exhibits extreme deformation for such experiments. We also found that for this specific case, the generalized neo-Hookean law predicts the experimental results better than the Skalak et al's law. Indeed this observation merits further detailed study on the role of different constitutive models, but that is not within the scope of the current study.

Once an appropriate form of $W_{s}$ is chosen, the principal elastic tensions (or stresses) in the cell membrane can be obtained as [69]

$$
\tau_{1}=\frac{1}{\epsilon_{2}} \frac{\partial W_{s}}{\partial \epsilon_{1}}, \quad \tau_{2}=\frac{1}{\epsilon_{1}} \frac{\partial W_{s}}{\partial \epsilon_{2}} .
$$

A finite element method (FEM) is used to obtain the membrane tensions resulting from the shear deformation and area dilatation $[30,31,34]$. In this approach, the surface of each 
cell is first discretized using Delauney triangles, as shown in figure 2, with each vertex (or node) shared by five or six triangles. The nodes also serve as the Lagrangian marker points that are advected to update the cell shape and location. Each triangle is assumed to remain flat upon deformation of the cell. The displacement field $\mathbf{v}$ is assumed to vary linearly within each triangular element, and is expressed in terms of linear shape functions $N_{i}$ as $\mathbf{v}=N_{i} \mathbf{v}_{i}$ where the index $i=0,1,2$ denotes the vertices of the triangle. The shape functions can be evaluated by knowing the coordinates of the vertices. Once the shape functions are known, the deformation gradient $\mathbf{F}$ is obtained. Subsequently, $\epsilon_{1}^{2}$ and $\epsilon_{2}^{2}$ which are the eigenvalues of $\mathbf{F} \cdot \mathbf{F}^{\mathrm{T}}$, and the principal stresses $\tau_{1}$ and $\tau_{2}$ are obtained. Having the stress tensor $\tau$ in each element evaluated, we obtain the resultant elastic force at each node as

$$
\mathbf{f}_{e}=\sum_{n} \int_{S_{n}} \frac{\partial \mathbf{N}}{\partial \mathbf{X}} \cdot \mathbf{P} \mathrm{d} S,
$$

where $\mathbf{N}$ is the vector of the shape functions, $\mathbf{P}=\epsilon_{1} \epsilon_{2} \tau \cdot \mathbf{F}^{-\mathrm{T}}$ is the first Piola-Kirchhoff stress tensor, and $S_{n}$ is the area of each of the $n$ triangles surrounding the node. Further $\mathrm{R} 1 \mathrm{C} 2$ details on the development of Eq. 4 can be found in our prior works [30,37].

The cell membrane also exerts a resistance against bending, and, hence formation of high curvature. The bending resistance is modeled following Helfrich's formulation for a bending energy that is expressed as [35]

$$
W_{b}=\frac{E_{b}}{2} \int_{S}\left(2 \kappa-c_{o}\right)^{2} d S
$$

where $E_{b} \sim 2-7 \times 10^{-19} \mathrm{~J}$ is the bending modulus, $\kappa$ is the mean curvature, $c_{o}$ is the spontaneous curvature, and $S$ is the entire surface area of a cell. An expression for a force density arising due to the bending resistance can be derived from Eq. 5 as

$$
\mathbf{f}_{b}=E_{b}\left[\left(2 \kappa+c_{o}\right)\left(2 \kappa^{2}-2 \kappa_{g}-c_{o} \kappa\right)+2 \Delta_{L B} \kappa\right] \mathbf{n}
$$

where $\kappa_{g}$ is the Gaussian curvature, $\Delta_{L B}$ is the Laplace-Beltrami operator, and $\mathbf{n}$ is the normal vector. The above force expression, which can be easily implemented within the 
IBM framework, is used here to model the bending resistance of the cell membrane. The mean and Gaussian curvatures are evaluated at each vertex using a quadratic surface fitted to the vertex and its nearest neighboring vertices. Then, using the Gauss theorem $\Delta_{L B} \kappa$ is approximated on a small surface patch $\mathrm{d} S$ as $(1 / \mathrm{d} S) \int_{l} \nabla_{S} \kappa \cdot \mathbf{n}_{l} \mathrm{~d} l$, where $l$ denotes the patch boundary, $\nabla_{S}$ the surface gradient, and $\mathbf{n}_{l}$ the unit normal to the boundary $l$. The gradient $\nabla_{S}$ on a surface triangle can be obtained either by a linear interpolation of the surface and $\kappa$, or using the loop subdivision method.

The net membrane force at each Lagrangian node on the cell surface is the summation of the contributions from shear, area dilation, and bending,

$$
\mathbf{f}_{m}=\mathbf{f}_{e}+\mathbf{f}_{b} .
$$

Further details of the numerical method dealing with blood cell deformation are given in our prior works $[30,36,37]$.

With regard to the mechanical properties of the membrane used in our simulations, we consider a membrane shear modulus $\left(G_{s}\right)$ of $2.5 \times 10^{-6} \frac{\mathrm{N}}{\mathrm{m}}$, membrane bending stiffness $\left(E_{b}\right)$ of $7 \times 10^{-19} \mathrm{~J}$, and fluid viscosities of $0.001 \mathrm{~Pa}-\mathrm{s}$, unless otherwise noted in the relevant section. With regard to the membrane consitutitive law, we use Eq. 1 with the exception of the micropipette aspiration simulations (Case III.C) where Eq. 2 is used. The membrane $\mathrm{R} 2 \mathrm{C} 4$ viscosity is assumed to be negligible in the present work. However, it has been incorporated within the present methodology in our previous study [37].

The white blood cells are modeled using the similar capsule model, and using the constitutive law given by Eq. 1, with the membrane stiffness assumed to be 10 times higher than the RBC. Furthermore, they are assumed to be non-nucleated. It should be noted that there are many different models of WBC that exist in the literature; for example: (1) liquid drop model without any membrane [70], (2) perfectly rigid sphere [71,72], and (3) 
capsule without any nucleus $[62,63]$. Our WBC model is similar to that of [62] and [63], and is sufficient for the purpose of the present study to demonstrate that we can consider diverse types of cells. Inclusion of one or multiple nuclei is a rather trivial issue within the front-tracking method, and we have previously published on WBC adhesion where we considered nucleated WBCs using the same numerical method as described here [64-66].

\section{C.2. Cell and flow coupling}

The cell membrane is deformed by the fluid flow; the membrane deformation in turn alters the flow. This two-way coupling between the membrane and the fluid is obtained via a continuous forcing IBM by adding the membrane force $\mathbf{f}_{m}$ to the governing equations for fluid flow and spreading the singular force over a finite span of the surrounding fluid using a delta function. This approach allows a single set of fluid flow equations written for both fluids, internal and external to the cell, and solved on a fixed Eulerian mesh (figure 1). For microcirculatory flows, inertial effects are negligible, and hence the flow is governed by the unsteady Stokes equations, along with the incompressibility condition:

$$
\begin{gathered}
\nabla \cdot \mathbf{u}=0 \\
\rho \frac{\partial \mathbf{u}}{\partial t}=-\nabla p+\nabla \cdot \mu\left[\nabla \mathbf{u}+\nabla \mathbf{u}^{\mathrm{T}}\right]+\mathbf{F}
\end{gathered}
$$

where

$$
\mathbf{F}=\int_{S} \mathbf{f}_{m} \delta\left(\mathbf{x}-\mathbf{x}^{\prime}\right) \mathrm{d} \mathbf{x}^{\prime}
$$

is the membrane force distributed to the surrounding fluid, $\delta$ is the three-dimensional Diracdelta function, and $\mathbf{x}$ and $\mathbf{x}^{\prime}$ are the locations in the flow domain and on the cell surface $S$, respectively [38]. Thus the delta function vanishes away from the cell membrane. Following [38], a cosine function spanning over four grid points around the cell boundary is used as a numerical approximation of the delta function

$$
\delta\left(\mathbf{x}-\mathbf{x}^{\prime}\right)=\frac{1}{64 \Delta^{3}} \prod_{i=1}^{3}\left[1+\cos \frac{\pi}{2 \Delta}\left(x_{i}-x_{i}^{\prime}\right)\right]
$$


where $\Delta$ is the Eulerian grid size. The method to solve the flow equations is described later. Once the flow field is obtained at any time instance, the velocity $\mathbf{u}_{m}$ of the marker points (Lagrangian nodes) on the cell surface is obtained by interpolating the Eulerian velocity $\mathbf{u}$ using the delta function as noted above. The membrane is then advected as $\mathrm{d} \mathbf{x}^{\prime} / \mathrm{d} t=\mathbf{u}_{m}$ to obtain the new deformed shape and position of the cells. It may be noted that $\mu$ in Eq. 9 is a function of $\mathbf{x}$ and time $t$ due to the presence of multiple fluids (i.e, plasma and cytoplasm). Thus $\mu$ has to be evolved with time as the flow field evolves. The method of evolving $\mu$ is described next in $\S I I-C .3$.

\section{C.3. Numerical treatment of viscosity}

We now discuss the method used to evolve the viscosity field $\mu(\mathbf{x}, t)$. As the deformable cells change shape and acquire new locations, the viscosity field must be evolved since the viscosity of the fluids interior and exterior to the cells are different. We follow the technique given in [38] in which $\mu(\mathbf{x}, t)$ is written in terms of an indicator function $I(\mathbf{x}, t)$ as

$$
\mu(\mathbf{x}, t)=\mu_{p}+\left(\mu_{c}-\mu_{p}\right) I(\mathbf{x}, t)
$$

where $\mu_{p}$ and $\mu_{c}$ are the viscosities of the plasma and cytoplasmic fluids. The indicator function is zero outside a cell and one inside. For the sake of brevity, we have assumed in Eq. 12 that only two fluids are present. However, the technique can be applied to any number of fluids, and, hence, to poly-disperse cell suspensions, by using multiple indicator functions. It can be shown that the indicator function follows a Poisson equation as

$$
\nabla^{2} I=\nabla \cdot \mathbf{G}
$$

where the $\mathbf{G}(\mathbf{x}, t)$ is an Eulerian variable constructed from the cell surface normals $\mathbf{n}$ as [38]

$$
\mathbf{G}(\mathbf{x}, t)=\int_{S} \delta\left(\mathbf{x}-\mathbf{x}^{\prime}\right) \mathbf{n} d S
$$

The delta function, as before, is numerically approximated by the cosine function given by Eq. 11. Thus the indicator function and the viscosity field vary smoothly across a cell 


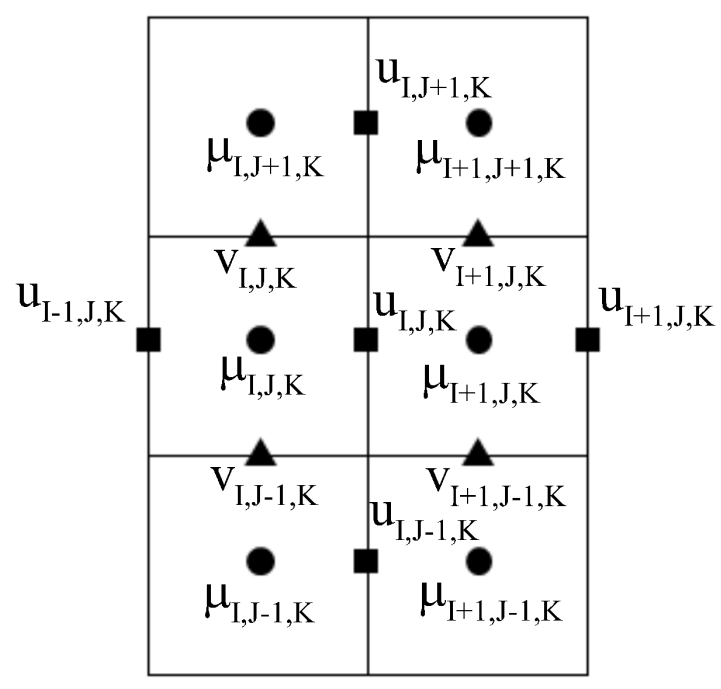

Figure 3: Stencil for viscosity $\mu(\mathbf{x})$.

membrane. Eq. 13 is solved to obtain $I$ at each time step, and update $\mu$. The solution procedure for Eq. 13 is similar to that of the Poisson equation for the projection operator $\phi$ as described in $\S I I-D .1$.

The presence of multiple fluids with different viscosity also requires care in dealing with the diffusion terms in Eq. 17. The natural choice is to treat the diffusion terms in conservation form, due to the finite-volume nature of the staggered-grid arrangement. For a constant viscosity field, the resulting discrete form is identical whether the diffusion terms are evaluated in conservation or non-conservation form. For a variable viscosity field, as in the present study, the conservative form is observed to significantly limit the time step size relative to the constant viscosity case. We find that this stability limit can be relaxed by using a non-conservation form of the variable viscosity terms. For example, for the diffusion terms in the $x$-component of the fluid momentum equation, we have

$$
\left\{\nabla \cdot\left[\mu\left(\nabla \mathbf{u}+\nabla \mathbf{u}^{\mathrm{T}}\right)\right]\right\}_{x}=\mu \nabla^{2} u+2 \frac{\partial \mu}{\partial x} \frac{\partial u}{\partial x}+\frac{\partial \mu}{\partial y}\left(\frac{\partial u}{\partial y}+\frac{\partial v}{\partial x}\right)+\frac{\partial \mu}{\partial z}\left(\frac{\partial u}{\partial z}+\frac{\partial w}{\partial x}\right)
$$


In the staggered-grid arrangement used here, $\mu(\mathbf{x}, t)$ is defined as cell-center based as shown in figure 3. Thus, an averaging scheme has to be used in evaluating the mixed derivative terms in Eq. 15. Consistent with typical staggered-grid averaging, we use

$$
\begin{aligned}
& \left.\frac{\partial \mu}{\partial x} \frac{\partial u}{\partial x}\right|_{I, J, K}=\left(\frac{\mu_{I+1, J, K}-\mu_{I, J, K}}{\Delta x}\right)\left(\frac{u_{I+1, J, K}-u_{I-1, J, K}}{2 \Delta x}\right) \\
& \left.\frac{\partial \mu}{\partial y} \frac{\partial u}{\partial y}\right|_{I, J, K}=\left(\frac{\mu_{I, J+1, K}+\mu_{I+1, J+1, K}-\mu_{I, J-1, K}-\mu_{I+1, J-1, K}}{4 \Delta y}\right)\left(\frac{u_{I, J+1, K}-u_{I, J-1, K}}{2 \Delta y}\right)
\end{aligned}
$$

$\left.\frac{\partial \mu}{\partial y} \frac{\partial v}{\partial x}\right|_{I, J, K}=\left(\frac{\mu_{I, J+1, K}+\mu_{I+1, J+1, K}-\mu_{I, J-1, K}-\mu_{I+1, J-1, K}}{4 \Delta y}\right)\left(\frac{v_{I+1, J, K}+v_{I+1, J-1, K}-v_{I, J, K}-v_{I, J-1, K}}{2 \Delta x}\right)$

and similarly for the z-direction. The indices I,J,K denote Eulerian grid points in the x-, y-, and z-directions, respectively.

\section{Modeling rigid interfaces}

We now discuss the methodology for simulating complex rigid interfaces, both stationary and moving, and the overall computational framework into which each of the distinct interface types are incorporated.

\section{D.1. Flow solver}

As noted in §II-C.2, the fluid flow is governed by the unsteady Stokes equations (Eq. 9). While there are several numerical methods available for a fast solution of the Stokes equations, here we follow an approach that is usually used for the full Navier-Stokes equations, and is very suitable for use with immersed boundary methods. In this approach, given the velocity and pressure fields at a time instance $n$, the governing equations are solved to advance the fields to the next time instance $n+1$, using a projection method. This method 
consists of first solving an advection-diffusion equation, and then solving a Poisson-type equation to enforce the conservation of mass. These steps can be represented in general form, as follows, treating the body force and off-diagonal viscous terms explicitly with a 2nd order Adams-Bashforth method, and the diagonal viscous terms semi-implicitly using the Crank-Nicholson method:

$$
\begin{gathered}
\rho \frac{u_{i}^{*}-u_{i}^{n}}{\Delta t}=G_{i}+\frac{3}{2} \alpha_{i}^{n}-\frac{1}{2} \alpha_{i}^{n-1}+\frac{1}{2}\left(\beta_{i}^{*}+\beta_{i}^{n}\right) \\
\frac{\partial^{2} \phi}{\partial x_{i} \partial x_{i}}=\frac{\rho}{\Delta t} \frac{\partial u_{i}^{*}}{\partial x_{i}} \\
\rho u_{i}^{n+1}=\rho u_{i}^{*}-\Delta t \frac{\partial \phi}{\partial x_{i}}
\end{gathered}
$$

where

$$
\begin{aligned}
\alpha_{i} & =F_{i}+\frac{\partial}{\partial x_{j}}\left(\mu \frac{\partial u_{j}}{\partial x_{i}}\right) \\
\beta_{i} & =\frac{\partial}{\partial x_{j}}\left(\mu \frac{\partial u_{i}}{\partial x_{j}}\right)
\end{aligned}
$$

Here we have assumed that the densities of the plasma and cytoplasmic fluids are the same, as is the case in blood flow, but their viscosities are different. The first step (Eq. 17) solves for an intermediate velocity field, $u_{i}^{*}$, that is in general non-solenoidal. The projection step (Eq. 19) corrects $u_{i}^{*}$ to be solendoial by projecting it onto a divergence-free space using a projection operator $\phi$ that is closely related to the pressure $p$. The variable $G_{i}$ in Eq. 17 is a placeholder for an approximation to the pressure gradient in the advection-diffusion step. Various authors have used different choices for $G_{i}$, as summarized in [42]. The choice for $G_{i}$ dictates the difference in magnitude between the intermediate velocity $u_{i}^{*}$ and the physical velocity, $u_{i}^{n+1}$, in addition to the relationship between $\phi$ and $p$. With projection methods the boundary conditions for the velocity are enforced at the advection-diffusion step on $u_{i}^{*}$, not $u_{i}^{n+1}$. The means of enforcing the boundary conditions is thus dependent on the choice of $G_{i}$, and the relevance of this to the present work is discussed shortly. The body force term computed from Eq. 10 representing the deformable interfaces enters the discrete governing equations as $F_{i}$ when solving Eq. 17-19. The complex rigid interfaces are 
simulated by enforcing specific constraints when solving these equations, as will be detailed later.

All spatial derivatives are evaluated using second-order differencing. For the viscous flows considered here, the semi-implicit treatment of the viscous terms in the advectiondiffusion equation is desirable for numerical stability. To take advantage of the fast inversion of tri-diagonal matrices, the advection-diffusion equation is solved using an Alternating Direction Implicit (ADI) scheme. The ADI scheme provides a robust means of solving Eq. 17 when compared with an iterative method where the performance can vary based on the complexity of the immersed boundary. The ADI scheme implemented here solves the advection-diffusion equation in four steps: The first step handles the explicit terms, while the remaining three steps handle the implicit sweeps in the $\mathrm{x}, \mathrm{y}$, and $\mathrm{z}$ directions, respectively.

In order to satisfy the incompressibility condition, the Poisson equation (Eq. 18) for $\phi$ must be solved implicitly. To attain a fast computation, we assume the computational domain to be periodic in one direction, and use the Fourier expansion. The 3D Poisson equation is then converted to a 2D problem in Fourier space which can be solved using fast, direct matrix inversion. Note that the periodic condition does not impose any limit on the geometric complexity of the immersed boundaries that can be considered.

\section{D.2. Complex geometry}

We now discuss the methodology used to treat rigid boundaries of arbitrary complexity. Figure 3(a) shows an example of a complex microvascular network that can be considered using the present methodology. The network is obtained from an in vivo image of a human cerebral microvasculature given in [39], and digitally rendered for use in the present context. 
(a)

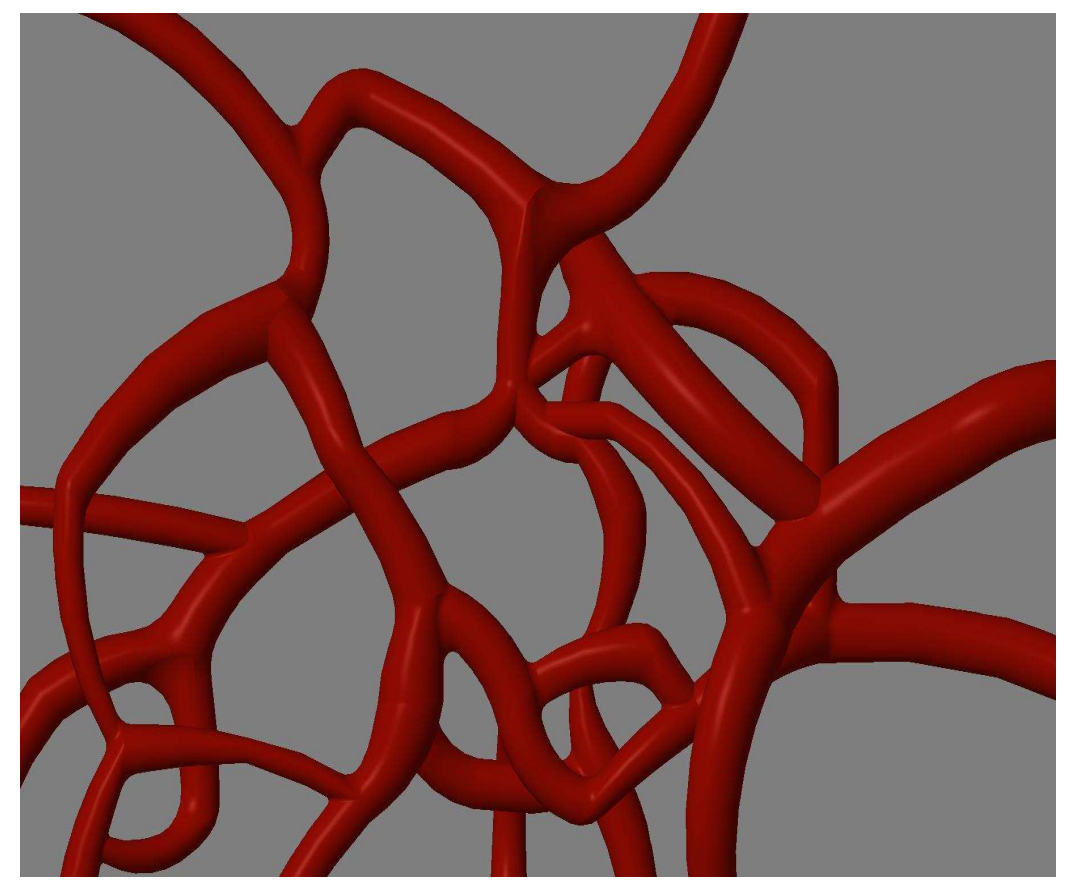

(b)

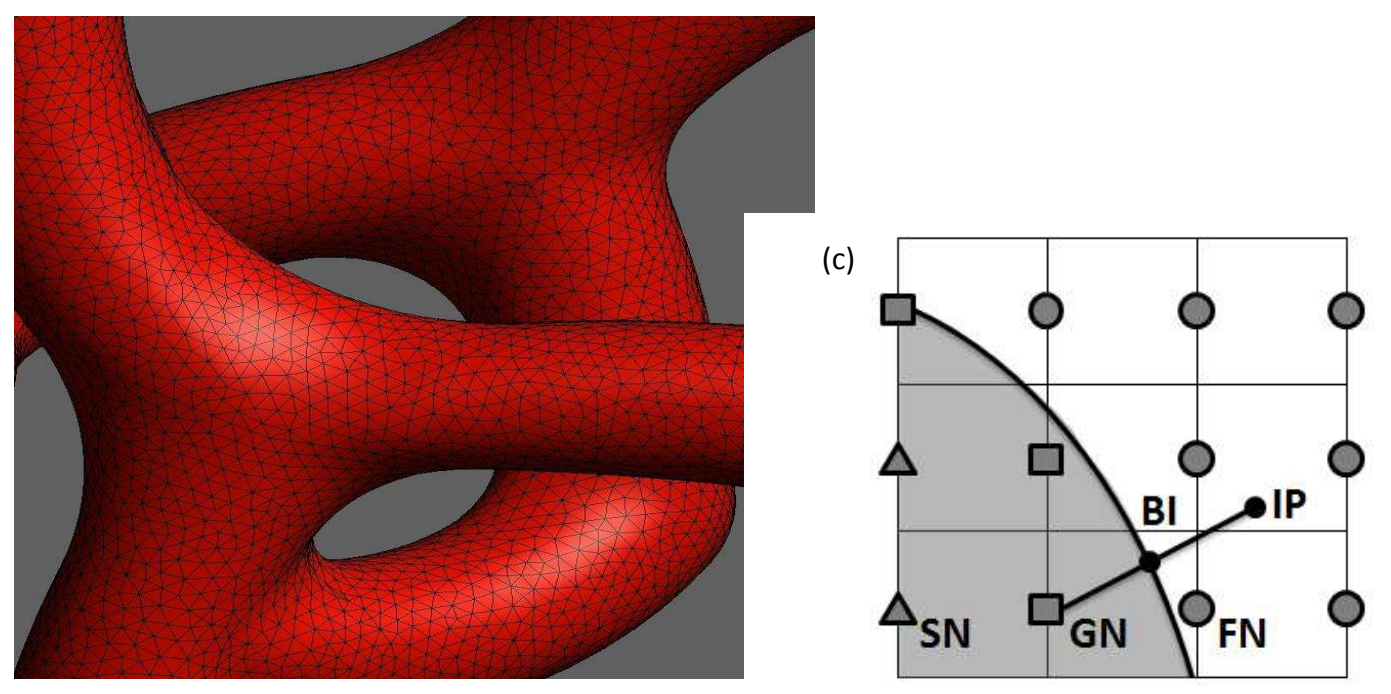

Figure 4: (a) An example of a complex geometry: a section of a microvascular network digitally rendered from an in vivo image of human cerebral microvasculature [39]. Blood vessels are shown in red. The network is characterized by highly tortuous, bifurcating and merging vessels. (b) A small part of the network is shown with the surface mesh on the vascular wall. (c) Computational stencil (for one velocity component) showing fluid nodes (FN, o), solid nodes (SN, $\Delta$ ), ghost nodes (GN, $\square$ ), boundary intercept (BI) and image point (IP). 
The walls of the vessels provide the boundaries for the fluid, and are taken to be stationary. These boundaries are treated as sharp-interface immersed boundaries. Following the in vivo image, the digital vascular network is first created using a standard CAD software package. The geometric data is then imported into the Gmsh mesh generating program [40] which is used to generate a triangular mesh on the vascular surface. The surface mesh is shown in figure 3(b) for a small section of the network. This surface mesh is then used to define the location of the sharp-interface boundary in the context of the GNIBM as described below. While the surface mesh approach provides a convenient means of defining complex surfaces, rigid boundaries of simpler geometry can also be represented analytically instead of using a surface mesh.

The GNIBM allows us to use a non-body conforming Eulerian mesh within which the fluid boundaries are embedded. In the present case, the entire domain encompassing all immersed boundaries is discretized using the same rectangular Cartesian mesh (figures 1 and 3c). A staggered arrangement of the variables on the Eulerian domain is utilized in which the velocity components are defined at the faces of a computational cell, and pressure is defined at the cell center. Figure 3(c) shows a computational stencil near an arbitrary rigid object interface. Once the boundaries of the rigid surface have been defined, the Eulerian nodes inside and outside the fluid domain are identified following the method in [41]. The nodes outside the fluid domain are identified as solid nodes (SN), and the solid nodes that have at least one neighboring fluid node are identified as ghost nodes (GN). After the GNs have been identified, the boundary-intercept (BI) point for each ghost node is determined by locating the nearest point on the rigid object surface. Following this, the image-points (IP) are determined for each GN, which are defined as the mirror image of the respective GN in the fluid domain, across the BI. Figure 3(c) illustrates the SN, GN, BI, and IP for an arbitrary rigid surface.

At the sharp-interface boundary, a no-slip condition has to be satisfied. The basic 
premise of the GNIBM is to enforce a velocity $\mathbf{u}_{G N}$ at the GN such that a known boundary condition $\mathbf{u}_{B I}$ is achieved at the BI. The value at the BI is taken to be the average of values at the GN and the IP. In general, the IPs do not coincide with the Eulerian nodes. A standard trilinear interpolant is used to obtain the velocity at the IP from the surrounding Eulerian nodes [41]. Thus, the boundary condition to be imposed at the GN becomes

$$
\mathbf{u}_{G N}=2 \mathbf{u}_{B I}-\sum_{m=1}^{8} \beta_{m} \mathbf{u}_{m}
$$

where $\beta_{m}$ are the weighing coefficients for the interpolation. The interpolation scheme results in a second-order accurate velocity field [41], and this is verified in §III-A for the present implementation.

As noted above, the no-slip condition has to be satisfied at the immersed boundary by enforcing the ghost node constraint (Eq. 20) when solving the advection-diffusion equation (Eq. 17). The approach we have developed to enforce this is now described. Implicitly imposing this constraint amounts to breaking the tri-diagonal structure of the matrices at the ADI steps. Therefore, we use the explicit form as

$$
\mathbf{u}_{G N}=2 \mathbf{u}_{B I}-\sum_{m=1}^{8} \beta_{m} \mathbf{u}_{m}^{n}
$$

where $\mathbf{u}^{n}$ is the known fluid velocity from the previous time level $n$. Since the ADI steps solve for the nonsolenoidal velocity $\mathbf{u}^{*}$, rather than the physical velocity, the boundary conditions must be expressed accordingly. In general, using Eq. 19 the nonsolenoidal velocity at a GN can be expressed in terms of the physical velocity as

$$
\mathbf{u}_{G N}^{*}=\mathbf{u}_{G N}+\Delta t \frac{\partial \phi}{\partial x_{i}}
$$

Then, the determination of $\mathbf{u}_{G N}^{*}$ reduces to the determination of the second term on the r.h.s. of the above equation. This term depends on the specific choice for $G_{i}$ in Eq. 17. The classical choices for $G_{i}$ are:

$$
\begin{aligned}
G_{i} & =0, \text { and } \\
G_{i} & =\frac{\partial p^{n}}{\partial x_{i}}
\end{aligned}
$$


For the choice of $G_{i}=0$, this term can be approximated as $\Delta t\left(\partial \phi^{n} / \partial x_{i}\right)+O\left(\Delta t^{2}\right)[43]$. It can be further shown that $\Delta t\left(\partial \phi^{n} / \partial x_{i}\right)=\mathbf{u}_{G N}^{*, n}-\mathbf{u}_{G N}^{n}$, where $\mathbf{u}_{G N}^{*, n}$ is the ${ }^{*}$ level value at a GN from the previous time step $n$. We use this form to express the condition at the immersed boundary. For the choice of $G_{i}=\partial p^{n} / \partial x_{i}$, it was shown in $[44,45]$ that $\Delta t\left(\partial \phi / \partial x_{i}\right)=O\left(\Delta t^{2}\right)$. Following this, the condition at the immersed boundary can be expressed as $\mathbf{u}_{G N}^{*} \approx \mathbf{u}_{G N}^{n}$. Our numerical experiments suggest that the use of either choice for $G_{i}$ results in negligible differences.

\section{D.3. Moving rigid bodies}

For moving rigid bodies of arbitrary shape, a method is developed to couple the rigidbody dynamics to the fluid flow. In this approach, the Euler parameters [46] are used as generalized coordinates to evolve the orientation of the object with time, while a Verlet algorithm [47] is used to integrate Newton's second law and determine the position of the object.

The translational acceleration a of a rigid object is obtained simply from Newton's second law $\mathbf{a}=\mathbf{F}_{0} / m$, where $\mathbf{F}_{0}$ is the net force exerted on the object by the surrounding fluid, and $m$ is the mass of the object. The position $\mathbf{x}_{0}$ and velocity $\mathbf{U}$ of the center of mass are computed by integrating $d \mathbf{U} / d t=\mathbf{a}$ and $d \mathbf{x}_{0} / d t=\mathbf{U}$. The method of computing $\mathbf{F}_{0}$ and the numerical integration to obtain $\mathbf{U}$ and $\mathbf{x}_{0}$ are described later.

The rotational motion of the object is governed by the conservation of angular momentum $\mathbf{H}$ as

$$
\mathbf{T}_{0}=\frac{d}{d t} \mathbf{H}
$$

where $\mathbf{T}_{0}$ is the torque exerted on the object by the surrounding fluid. The angular velocity 
$\omega$ is

$$
\omega=\mathbf{I}_{0}^{-1} \cdot \mathbf{H}
$$

where $\mathbf{I}_{0}$ is the moment of inertia tensor. Note that Eq. 25 is written in a space-fixed coordinate system, whereas Eq. 26 is in the body-fixed system. The basis vectors in the two coordinate systems are related via a rotation matrix $\mathbf{R}_{e}$ which can be expressed completely in terms of the four Euler parameters [46]. The Euler parameters are defined as

$$
\begin{aligned}
e_{0} & =\cos \frac{\beta}{2} \\
{\left[\begin{array}{l}
e_{1} \\
e_{2} \\
e_{3}
\end{array}\right] } & =\mathbf{n} \sin \frac{\beta}{2}
\end{aligned}
$$

where $\beta$ represents a finite rotation about an arbitrary axis $\mathbf{n}$. The angular velocity can then be written as

$$
\omega=\mathbf{I}_{0}^{-1} \cdot\left(\mathbf{R}_{e} \cdot \mathbf{H}\right)
$$

The Euler parameters are used as generalized coordinates to evolve the orientation of the object with time. In terms of the Euler parameters, the rotational equations of motion are:

$$
\left[\begin{array}{c}
\dot{e_{0}} \\
\dot{e_{1}} \\
\dot{e_{2}} \\
\dot{e_{3}}
\end{array}\right]=0.5\left[\begin{array}{cccc}
e_{0} & -e_{1} & -e_{2} & -e_{3} \\
e_{1} & e_{0} & -e_{3} & e_{2} \\
e_{2} & e_{3} & e_{0} & -e_{1} \\
e_{3} & -e_{2} & e_{1} & e_{0}
\end{array}\right]\left[\begin{array}{c}
0 \\
\omega_{1} \\
\omega_{2} \\
\omega_{3}
\end{array}\right]
$$

where $\omega_{1}, \omega_{2}, \omega_{3}$ are the angular velocity components determined by Eq. 28 . The numerical integration of Eq. 29 is described later.

To numerically compute the force $\mathbf{F}_{0}$ and torque $\mathbf{T}_{0}$, the object surface is discretized using triangular elements similar to that used for the deformable cells (figure 2). At each surface element, the fluid stress tensor is computed from the known velocity and pressure of the surrounding fluid. The pressure is obtained from the projection operator $\phi$ which was introduced before in the context of Eq. 17. As mentioned there, the relationship between $p$ and $\phi$ depends on the choice of $G_{i}$. For the specific choices of $G_{i}$ used here as given by 
Eqs. 23 and 24, the respective equations for pressure become $[42,48]$ :

$$
\begin{aligned}
p & =\phi-\frac{1}{2} \frac{\partial u_{i}^{*}}{\partial x_{i}}, \quad \text { and } \\
p^{n+1} & =p^{n}+\phi-\frac{1}{2} \frac{\partial u_{i}^{*}}{\partial x_{i}} .
\end{aligned}
$$

Once the pressure field is known, the pressure at each surface element is computed by interpolating from the surrounding Eulerian nodes. For computing the viscous stresses at the object surface, the velocity derivatives are evaluated using second-order differencing. A three-point stencil is used with one end point located on the object surface and the other two points in the fluid domain. The velocity at the surface point is computed from the translational and rotational velocities of the object, while those at the other two points are interpolated from the surrounding Eulerian nodes. The computation of the surface stress tensor can be simplified if the object has an analytically defined shape, such as an ellipsoid. Since the rigid objects considered here are either spherical particles or platelets that have an oblate spheroidal shape, we use either the spherical or the oblate spheroidal coordinate system, respectively. The major benefit to using such a coordinate system is that it minimizes both the amount of computation and number of interpolations. Furthermore, it permits a direct analytical evaluation of some of the velocity derivative terms. Once the stress tensor at each surface element is known, the net force and torque acting on the object can be readily obtained. The force and torque are then used to evolve the position and orientation of the object.

A Verlet algorithm [47] is used to update the position and translational velocity of the object as

$$
\begin{aligned}
& \mathbf{x}_{0}(t+\Delta t)=\mathbf{x}_{0}(\Delta t)+\Delta t \mathbf{U}(t)+0.5 \mathbf{a}(t) \Delta t^{2} \\
& \mathbf{U}(t+\Delta t)=\mathbf{U}(t)+\frac{1}{2} \Delta t[\mathbf{a}(t)+\mathbf{a}(t+\Delta t)]
\end{aligned}
$$

For the angular momentum, a leapfrog integration scheme is used as follows:

$$
\mathbf{H}(t)=\mathbf{H}(t-0.5 \Delta t)+0.5 \Delta t \mathbf{T}_{0}(t)
$$




$$
\begin{gathered}
\mathbf{e}(t+0.5 \Delta t)=\mathbf{e}(t)+0.5 \Delta t \dot{\mathbf{e}}(t) \\
\mathbf{H}(t+0.5 \Delta t)=\mathbf{H}(t-0.5 \Delta t)+\Delta t \mathbf{T}_{0}(t) \\
\omega(t+0.5 \Delta t)=\mathbf{I}_{0}^{-1}\left[\mathbf{R}_{e}(t+0.5 \Delta t) \mathbf{H}(t+0.5 \Delta t)\right] \\
\mathbf{e}(t+\Delta t)=\mathbf{e}(t)+\Delta t \dot{\mathbf{e}}(t+0.5 \Delta t) \\
\omega(t+\Delta t)=\mathbf{I}_{0}^{-1}\left[\mathbf{R}_{e}(t+\Delta t) \mathbf{H}(t+\Delta t)\right]
\end{gathered}
$$

Thus, at $t+\Delta t$, the new position, orientation, and translational and angular velocities of the object are obtained. These are then used in turn to apply the appropriate boundary conditions on the object surface (i.e. moving sharp-interface immersed boundary) as discussed in $\S I I-D .2$.

\section{Validation}

A series of validation studies is considered to establish the accuracy of the methodology. These validation studies involve stationary rigid objects, moving rigid objects, and simulations that involve both complex rigid boundaries and deformable cells. Each of the cases was chosen to both demonstrate and test specific aspects of the methodology, as highlighted in Table I and discussed in the relevant sections. Results are compared with analytical solutions, experimental studies, and previously published numerical results. Since our interest is in blood cell motion for which the inertial effect is negligible, we limit our validation studies to Stokes flow. It should be noted that validation of the deformable cell model in simple geometry had been considered in our previous studies, e.g., [30,36,37,49]. 
(a)

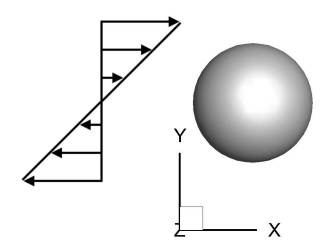

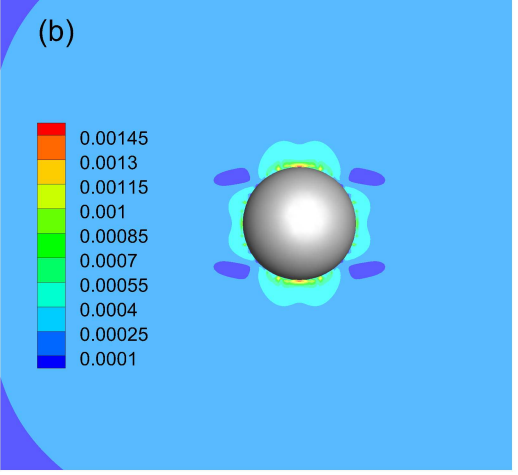

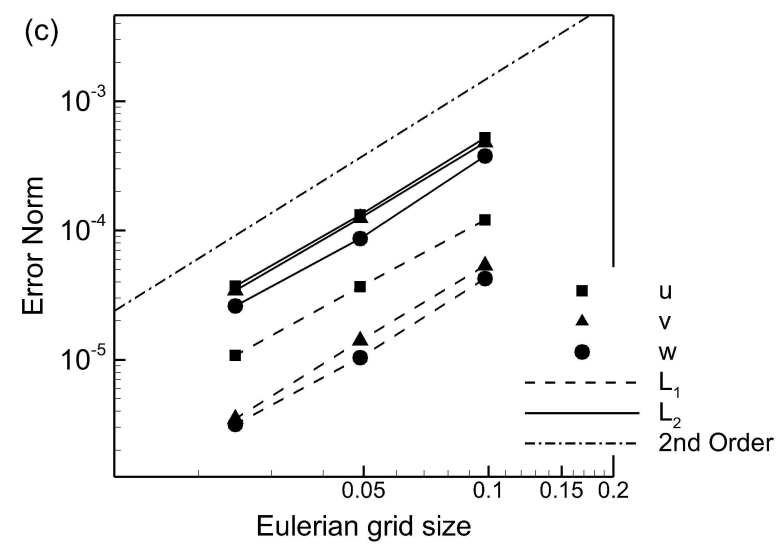

Figure 5: Validation for stationary rigid body: (a) Schematic of a fixed rigid sphere subjected to a simple shear flow. (b) $L_{\infty}$ error norm of the velocity magnitude for $256^{3}$ Eulerian resolution with respect to the analytical solution. (c) $L_{1}$ and $L_{2}$ error norms as functions of Eulerian resolution varied as $64^{3}, 128^{3}$ and $256^{3}$. 


\begin{tabular}{|c|l|l|}
\hline Sub-Section & Description & Purpose \\
\hline \hline A & $\begin{array}{l}\text { Stationary rigid sphere } \\
\text { in a shear flow }\end{array}$ & $\begin{array}{l}\text { Verify the accuracy order for the rigid interface } \\
\text { methodology; Establish a mesh resolution crite- } \\
\text { ria for rigid interfaces }\end{array}$ \\
\hline B.1 & $\begin{array}{l}\text { Rotating spheroid in a } \\
\text { shear flow }\end{array}$ & $\begin{array}{l}\text { Establish numerical accuracy for moving rigid } \\
\text { bodies }\end{array}$ \\
\hline B.2 & $\begin{array}{l}\text { Sphere moving near a } \\
\text { wall }\end{array}$ & $\begin{array}{l}\text { Establish numerical accuracy for moving and } \\
\text { non-moving rigid boundaries in close proximity }\end{array}$ \\
\hline C & $\begin{array}{l}\text { Micropipette aspiration } \\
\text { of a red blood cell }\end{array}$ & $\begin{array}{l}\text { Compare numerical results with experimental } \\
\text { data for one cell undergoing extreme deforma- } \\
\text { tion in proximity to a rigid boundary }\end{array}$ \\
\hline D & $\begin{array}{l}\text { Multiple deformable } \\
\text { RBCs in complex } \\
\text { geometry }\end{array}$ & $\begin{array}{l}\text { Compare numerical results with experimental } \\
\text { data for high hematocrit flows in complex ge- } \\
\text { ometry }\end{array}$ \\
\hline E & $\begin{array}{l}\text { Flow of a capsule } \\
\text { through a constricted } \\
\text { channel }\end{array}$ & $\begin{array}{l}\text { Compare our results with that achieved using an } \\
\text { alternate numerical method }\end{array}$ \\
\hline
\end{tabular}

Table I: Specific objectives of each of the validation studies (R2C2)

\section{A. Stationary rigid objects}

In our first validation study we demonstrate the accuracy of the methodology for stationary rigid objects. We choose the classical problem of a sphere subjected to a linear shear flow given by

$$
\mathbf{u}^{\infty}=[\dot{\gamma} y, 0,0]
$$

where $\dot{\gamma}$ is the shear rate. The set up is schematically shown in figure $5(\mathrm{a})$. The sphere is not allowed to translate and rotate. An analytical solution for this problem for an unbounded domain and in the absence of inertia can be derived as

$$
\begin{aligned}
& u=\frac{y \dot{\gamma}}{2}\left[1-\left(\frac{r}{a}\right)^{-5}\right]+\frac{y \dot{\gamma}}{2}\left[1-\left(\frac{r}{a}\right)^{-3}\right]-\frac{5}{2}\left(\frac{x}{a}\right)^{2} y \dot{\gamma}\left[\left(\frac{r}{a}\right)^{-5}-\left(\frac{r}{a}\right)^{-7}\right] \\
& v=\frac{x \dot{\gamma}}{2}\left[1-\left(\frac{r}{a}\right)^{-5}\right]-\frac{x \dot{\gamma}}{2}\left[1-\left(\frac{r}{a}\right)^{-3}\right]-\frac{5}{2}\left(\frac{y}{a}\right)^{2} x \dot{\gamma}\left[\left(\frac{r}{a}\right)^{-5}-\left(\frac{r}{a}\right)^{-7}\right] \\
& w=-\frac{5}{2} \frac{x y z \dot{\gamma}}{a^{2}}\left[\left(\frac{r}{a}\right)^{-5}-\left(\frac{r}{a}\right)^{-7}\right]
\end{aligned}
$$


where $r=\sqrt{x^{2}+y^{2}+z^{2}}$ and $a$ is the sphere radius. The numerical results will be compared with this analytical solution.

In our simulation the sphere is represented as a sharp-interface immersed boundary, and in this context the sphere surface is defined by an analytical function. A cubic computational domain is considered which is discretized by a rectangular Cartesian mesh. The domain edge length is taken to be $12 a$. The Eulerian resolution is varied as $64^{3}, 128^{3}$, and $256^{3}$. The boundary conditions applied at the $x$ and $y$ boundaries are the analytical solution given by Eq. 40, and the condition applied at the $z$ boundaries is the periodic condition. The domain edge length considered here was found to be sufficient to eliminate any boundary effect.

Figure 5(b) shows contours of the $L_{\infty}$ error norm of the numerical solution at $256^{3}$ resolution with respect to the analytical solution based on the velocity magnitude. The numerical result agrees very well with the analytical one as the maximum $L_{\infty}$ norm is $\sim 0.001$. As can be seen in the figure, the maximum error occurs near the sphere surface and decreases with increasing distance from the surface, a trend that is expected in an IBM. Figure 5(c) shows the $L_{1}$ and $L_{2}$ error norms of the three velocity components as functions of the Eulerian resolution. A second-order trend is evident here, providing a measure of the global accuracy of the method. We use the results of this section to establish a guideline for determining the Eulerian resolution to use with arbitrarily complex geometries.

With the sharp-interface method, the degree to which an object surface is resolved is directly controlled by the Eulerian resolution; a greater number of grid points, and hence ghost nodes, will result in a better representation of the surface curvature. For the present case of a stationary sphere we quantify the degree to which the surface is represented by the number of Eulerian points across the sphere diameter. As the Eulerian resolution is 
increased from $128^{3}$ to $256^{3}$, the number of grid points across the sphere diameter changes from $\sim 20$ to $\sim 40$, and the maximum $L_{\infty}$ norm changes from $\sim 0.004$ to $\sim 0.001$. Thus $20-$

40 grid points across the sphere diameter keep the error within a reasonable limit. For the R1C6 R1C6 sphere, the number of Eulerian points across the diameter is a convenient metric for quantifying the resolution of the surface in terms of Eulerian mesh. For spheroidal geometries, R1C5 we use this criteria in conjunction with the equivalent spheroid diameter in determining the appropriate resolution, as discussed later. For arbitrarily complex surfaces, this guideline is extended by examining the regions of highest curvature and selecting an appropriate Eulerian resolution in accordance. For complex microvascular networks, for example, the means by which we extend this approach is two-fold. First, we look at the minimum vessel diameter and ensure that the number of grid points across is in accordance with the criteria. Second, we examine the surface curvature relative to the Eulerian resolution. We extend a ray across the regions where curvature is highest, and check that the number of Eulerian grid points per unit length is also in accordance with the established criteria. In general, this criteria is used when selecting the Eulerian resolution to be used with the subsequent validation cases, and thus its use is verified by the accuracy of the results.

\section{B. Moving rigid body}

We now validate the accuracy of the methodology for moving rigid boundaries by considering two classical problems as follows.

\section{B.1. Spheroid in shear flow}

The first example is the unsteady motion of an oblate spheroid in a linear shear flow. The set up is schematically shown in figure 6(a). The imposed linear shear flow is given by Eq. 
(a)

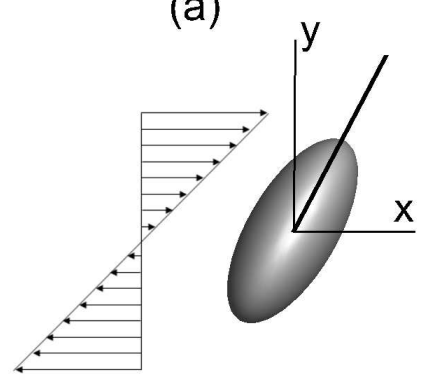

(b)

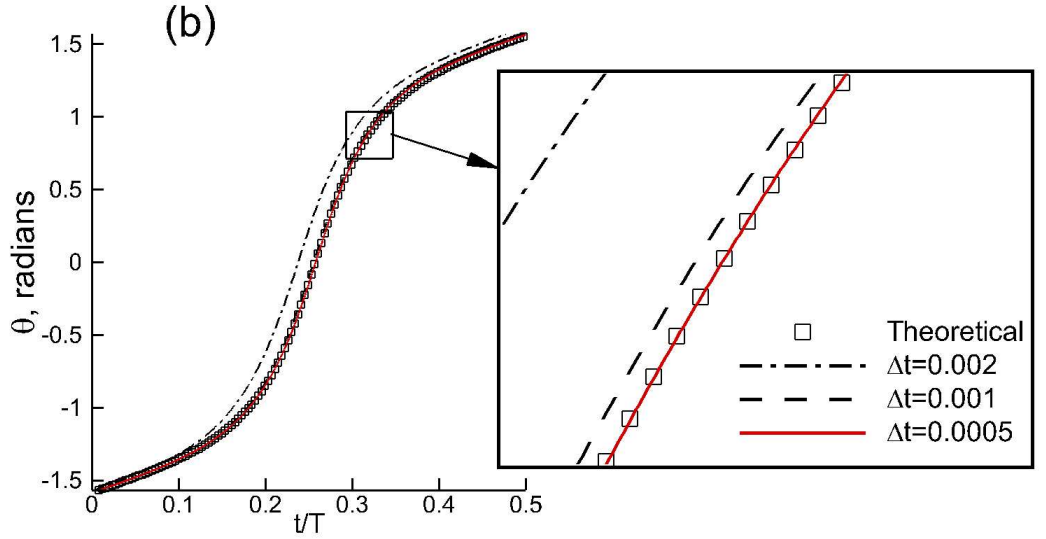

Figure 6: Validation for moving rigid body: (a) Schematic of a rigid oblate spheroid undergoing flipping motion when subjected to a simple shear flow. (b) Instantaneous orientation angle $\theta(t)$ is shown over one half period of flipping for the analytical solution ( $\square$ ) of Jeffery [50], and numerical solutions for different time steps $\dot{\gamma} \Delta t=0.002(---), 0.001\left(--^{-}\right)$, and $0.0005(-)$. A close-up is shown in the inset. Here $\alpha=3$.

39. The oblate spheroid is free to translate and rotate by the action of the force and torque exerted on it by the surrounding fluid. We place the center of the spheroid at the origin of the coordinate system so that there is no relative translational motion between the fluid and the spheroid. However, under the fluid torque, the spheroid executes an unsteady flipping motion in which its orientation changes with time. As before, we assume that inertial effects are negligible. In this limit, and with further assumption of an unbounded fluid domain, an exact analytical solution [50] describing the spheroid motion can be expressed as

$$
\theta(t)=\tan ^{-1}\left(\alpha \tan \left[\frac{t \dot{\gamma}}{\alpha+\frac{1}{\alpha}}\right]\right)
$$

where $\theta$ is the instantaneous inclination angle of the spheroid's major axis, and $\alpha$ is the ratio of the major axis to minor axis lengths. This exact solution is used to validate our immersed-boundary simulation results.

In the numerical simulation, we consider an oblate spheroid of aspect ratio $\alpha=3$. While the object surface can be defined analytically, a surface Lagrangian mesh is needed 
for force and torque computation. We use 1,280 surface triangles for this purpose which was observed to be sufficient after testing with various surface resolutions. While using a relatively coarse surface resolution obviously results in the surface curvature not being adequately represented, using an excessively fine mesh resolution results in an increased number of interpolations to be performed at each surface element. In general it was observed that a mesh resolution resulting in an average element side length on the same order of the Eulerian grid spacing was a good criterion for selecting a surface resolution. As noted in $\S I I-D .3$, an oblate spheroidal coordinate system is used for efficient computation of force and torque. A cubic computational domain with edge length $L=12 r_{s p}$ is considered where $r_{s p}$ is the radius of a sphere having the same volume as that of the spheroid. The Eulerian resolution used is $160^{3}$, resulting in a resolution in accordance with the aforementioned criteria, based on the equivalent spheroid diameter. The domain is periodic in the $x$ - and $z$-directions, and wall bounded in the $y$-direction. The influence of the domain size was tested, and $L=12 r_{s p}$ is found to have no significant effect due to finite domain size.

The simulation result for inclination angle $\theta(t)$ is presented in figure $6(\mathrm{~b})$ over one half period of flipping for $\alpha=3$, and for three different time step sizes, namely $\dot{\gamma} \Delta t=$ 0.002, 0.001, and 0.0005. An excellent agreement with Eq. 41 is observed as $\Delta t$ is decreased. The maximum absolute error in $\theta(t)$ over one-half period for each of the time step sizes is $0.4,0.052$, and 0.014 radians, respectively, while the $L_{2}$ error norms are $0.167,0.0257$, 0.00755 , respectively.

\section{B.2. Sphere moving near a wall}

The next validation study considered for moving bodies is the motion of a sphere in a linear shear flow near a stationary plane wall as shown in figure 7(a). The sphere is free to translate and rotate by the action of the fluid force and torque. In the absence of inertia, 
the sphere moves parallel to the wall with constant translational and angular velocities. The translation and angular velocities decrease with decreasing distance between the sphere and the wall. Analytical solutions have been developed for this problem in the limit of negligible inertia [51,52]. These classical solutions typically express the sphere's translational velocity $U$ and the angular velocity $\Omega$ in terms of expansions in $a / H$, where $a$ is the sphere radius and $H$ is the distance of the sphere center from the wall. A Pade approximation to the classical solution was obtained as

$$
\begin{aligned}
& U^{*}=\frac{U}{U_{0}}=\left[\sum_{i=0}^{37} q_{i}^{(U)}\left(\frac{a}{H}\right)^{i}\right] /\left[\sum_{i=0}^{37} r_{i}^{(U)}\left(\frac{a}{H}\right)^{i}\right] \\
& \Omega^{*}=\frac{\Omega}{\Omega_{0}}=\left[\sum_{i=0}^{37} q_{i}^{(\Omega)}\left(\frac{a}{H}\right)^{i}\right] /\left[\sum_{i=0}^{37} r_{i}^{(\Omega)}\left(\frac{a}{H}\right)^{i}\right]
\end{aligned}
$$

where $U_{0}$ and $\Omega_{0}$ are the values in the absence of the wall, and $q_{i}$ and $r_{i}$ are the coefficients in the expansions [53]. We validate our numerical results against Eqs. 42 and 43.

Both the moving sphere and the stationary wall are simulated as sharp-interface immersed boundaries. The minimum distance between the sphere surface and the wall considered in the simulations is as small as $<6 \%$ of the sphere diameter. Thus, this validation study establishes the accuracy of the methodology when both moving and stationary immersed boundaries are in close proximity. In order to calculate the necessary parameters for the ghost-node identifications, the sphere surface is represented analytically. However, for the purpose of force and torque computation, the surface is discretized by 1,280 triangles. The computational domain is cubic with edge length $13 a$, and the Eulerian resolution used is $160^{3}$.

Simulations are performed for different values of $a / H$ in the range of 0.3 to 0.9 . The numerical results for the dimensionless translational and angular velocities, $U^{*}$ and $\Omega^{*}$, are compared with the analytical ones in figure 7(b). All 38 terms in the analytical expression were used. In general, the two solutions agree very well as presented in the figure, even 
(a)
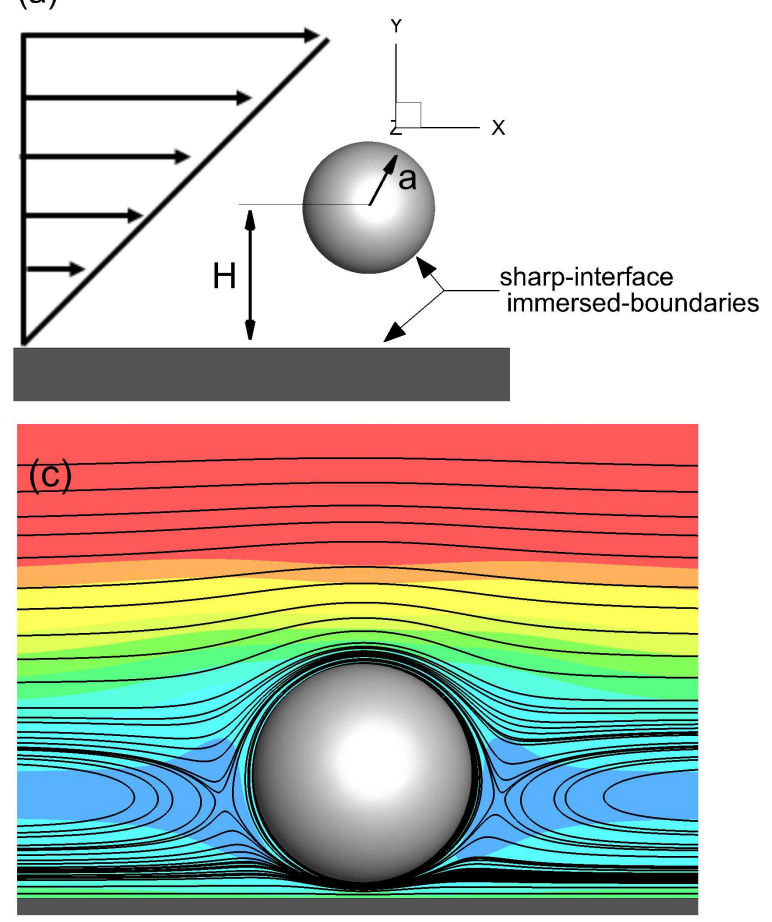

(b)

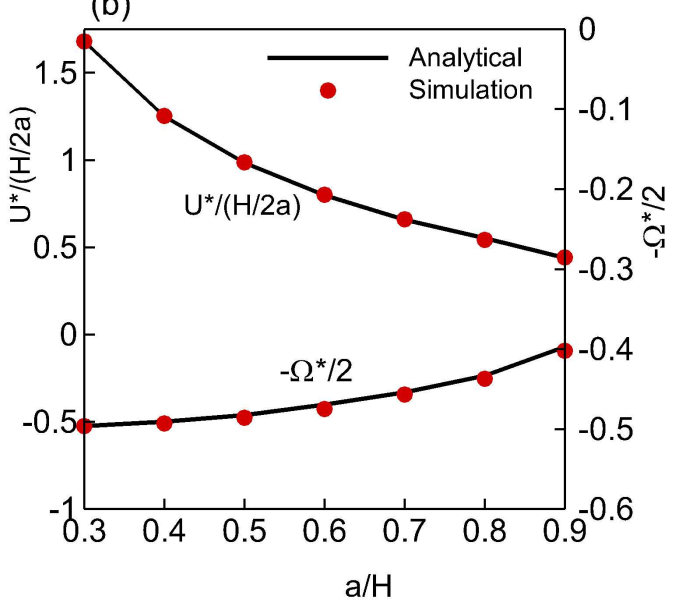

Figure 7: Validation for moving rigid objects: (a) Schematic of a sphere freely moving and rotating in a shear flow near a stationary wall; (b) Comparison of numerical simulation $(\bullet)$ and analytical result $(-)$ for dimensionless translational $\left(U^{*}\right)$ and rotational $\left(\Omega^{*}\right)$ velocities as functions of $a / H$. Note that $U^{*}$ and $\Omega^{*}$ are scaled as noted in the plot. (c) Contours of velocity magnitude (range 0 to 1 ) and streamlines for $a / H=0.9$. 
for $a / H=0.9$ for which there are three Eulerian points between the sphere surface and the immersed wall. The absolute error for $U^{*}$ and $\Omega^{*}$ ranges from 0.00027 to 0.0037 , and 0.00037 to 0.0046 , respectively, as $a / H$ is increased from 0.3 to 0.9 . One reason why error increases at higher values of $a / H$ is that the analytical solution itself becomes inaccurate as $a / H$ becomes large.

Figure $7(\mathrm{c})$ provides contours of the velocity magnitude and streamlines in the frame of of reference of the sphere for $a / H=0.9$. As can be seen here, flow variables vary smoothly around the sphere and near the wall. Consistent with the characteristics of Stokes flow, the flow field exhibits fore-aft symmetry. Similar smoothness in the flow field and symmetry in accordance with Stokes flow were verified for the validation studies presented earlier.

\section{Micropipette aspiration of a red blood cell}

The next validation study deals with a deformable interface in the presence of a complex stationary boundary. Specifically, we simulate the experiment of [54] in which an RBC was aspirated into a micropipette by applying a pressure difference $\Delta P$. The RBC exhibited a large deformation as it was partly drawn into the pipette, and the length $L$ of the RBC inside the pipette was measured as a function of $\Delta P$. The purpose of this case is to

demonstrate, in a quantitative manner, the accuracy of the methodology in capturing the extreme deformation of an individual cell, in addition to the short range interaction between the two types of interfaces. This is one case for which reliable experimental results exist, and thus provides a good benchmark for the accuracy of the method.

The setup for our simulation is shown in figure 8(a), where the RBC and micropipette are immersed in a viscous fluid. The pipette itself is a cylinder of small but finite thickness 

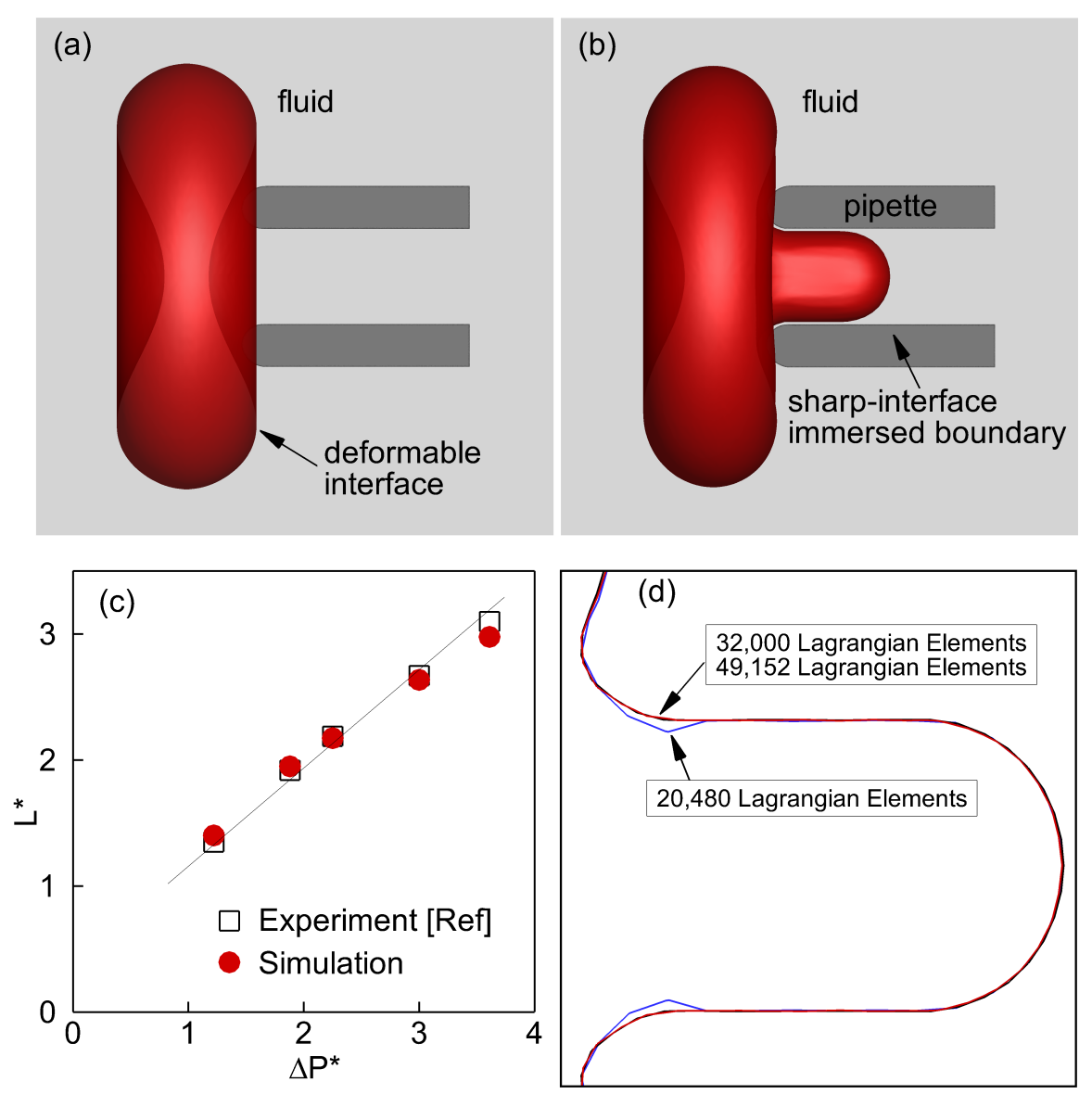

Figure 8: Validation for interaction between deformable and rigid interfaces: Micropipette aspiration of an RBC. (a) The undeformed RBC (red) at the beginning of aspiration. The pipette is modeled as a cylinder of finite thickness (grey). Both the cell and the pipette are immersed in a viscous fluid. (b) Snapshot from the simulation after the steady state is reached. A portion of the cell is drawn in to the pipette. (c) Comparison of the present simulation results $(\bullet)$ and experimental data $(\square,[54])$. Here $L^{*}=L / r_{p}$ is the dimensionless length of the cell drawn inside the pipette, and $\Delta P^{*}=\Delta p\left(r_{p} / 2 G_{s}\right)$ is the dimensionless pressure, where $r_{p}$ is the inside radius of the pipette. (d) Sensitivity of the results to the Lagrangian resolution on the RBC surface. $2 \mathrm{D}$ contours of the cell volume residing inside the pipette are shown for three different Lagrangian resolutions by varying the number of surface elements as $20480(-), 32000(-)$, and $49152(-)$. 
with a rounded inlet edge. Numerically it is modeled as a rigid object using the GNIBM. The inside diameter is $1.8 \mu \mathrm{m}$ with a thickness of $0.8 \mu \mathrm{m}$, while the length is $4.3 \mu \mathrm{m}$. The R1C8 computational domain into which it is immersed has dimensions $8.6 \mu \mathrm{m} \times 11.5 \mu \mathrm{m} \times 11.5$ $\mu \mathrm{m}$, in the $\mathrm{x}_{-}, \mathrm{y}_{-}$, and $\mathrm{z}$-directions, respectively, and the cylinder axis is oriented in the $\mathrm{x}$-direction. The outlet face of the cylinder coincides with the outlet of the computational domain, and pressure boundary conditions are applied at the bounds of the domain in the $\mathrm{x}$-direction in accordance with the applied pressure difference. The domain is wall bounded in the $\mathrm{y}$-direction and periodic in the z-direction, with lengths taken so as to eliminate any effect on the result. The deformable RBC is modeled using the FEM as described in $§ I I-$ C.1, with the membrane strain energy modeled by Eq. 2. As the deformation of the RBC is strongly influenced by the micropipette geometry, this problem involves a close interaction between the deformable cell membrane and the sharp-interface rigid boundary.

Figures $8(\mathrm{a})$ and (b) provide snapshots from a simulation at the beginning and at a later time after a steady state has been reached. The initial shape of the RBC at the beginning of the simulation is the biconcave discocyte. With the applied pressure, a part of the cell volume begins entering the pipette. Eventually a steady state is reached under the balance of the applied pressure and the tension generated in the cell membrane. The large deformation and an extended length of the cell inside the pipette is evident in figure 8(b). Also noteworthy is the smoothness of the RBC surface even after the extreme deformation. The numerical results are compared with the experimental data of [54] in figure 8(c) where the cell length $L$ inside the pipette is plotted as a function of the applied pressure difference $\Delta P$. Good agreement between the two studies is observed. It should be noted that the results are sensitive to the the actual values of the model parameters used for the RBC membrane. For the numerical data shown here we used $G_{s}=7 \times 10^{-6} \mathrm{~N} / \mathrm{m}$ as noted in [54], and $C_{3}=G_{s} / 30$ as noted in [33]. Also discussed in [33] is that use of Eq. 2 is well R2C6 suited for matching experimental data for large deformation, and provides a much better match with experiments using this equation over lower order ones of the same type. With 
regard to the common types of strain energy model typically utilized in numerical methods, for this case we have found that Eq. 2 better predicts the experimental results than Eq. 1.

Sensitivity of the results to the Lagrangian resolution on the surface of the $\mathrm{RBC}$ is shown in figure $8(d)$ where $2 \mathrm{D}$ contours of the cell volume residing inside the pipette are shown. The resolution criteria for deformable cells has been established in our prior works $[30,36]$. R1C5 The general criteria is that the average side length of an element of the Lagrangian mesh is on the same order as the spacing of the Eulerian mesh. Simulations are performed with three different Lagrangian resolutions, namely 20480, 32000, and 49152 triangular elements on the RBC surface. While the shape obtained with 20480 elements lacks smoothness at the pipette entrance, those with 32000 and 49152 elements are smooth and indistinguishable. The sensitivity to the Eulerian resolution is also tested using $96 \times 128 \times 128$ and $192 \times 256 \times 256$ grids, and the difference was observed to be negligible.

\section{Multiple deformable RBCs in complex geometry}

The next validation study considers multiple deformable interfaces in the presence of nonmoving rigid boundaries. The specific example considered is the flow of multiple deformable RBCs in a bifurcating and merging rectangular channel (figure 9). It is well known in microcirculatory physiology that when a suspension of RBCs reaches a vascular bifurcation, it does not split in proportion to the flow rates in the two daughter branches. Rather, the branch with the higher flow rate gets a disproportionately greater number of cells. This phenomenon, known as the Zweifach-Fung (Z-F) effect, is believed to be one reason why blood cell distribution is not uniform across different vessels in microcirculation [4-6]. In this section we simulate the Z-F effect and compare our numerical results against published experimental results. 
(a)
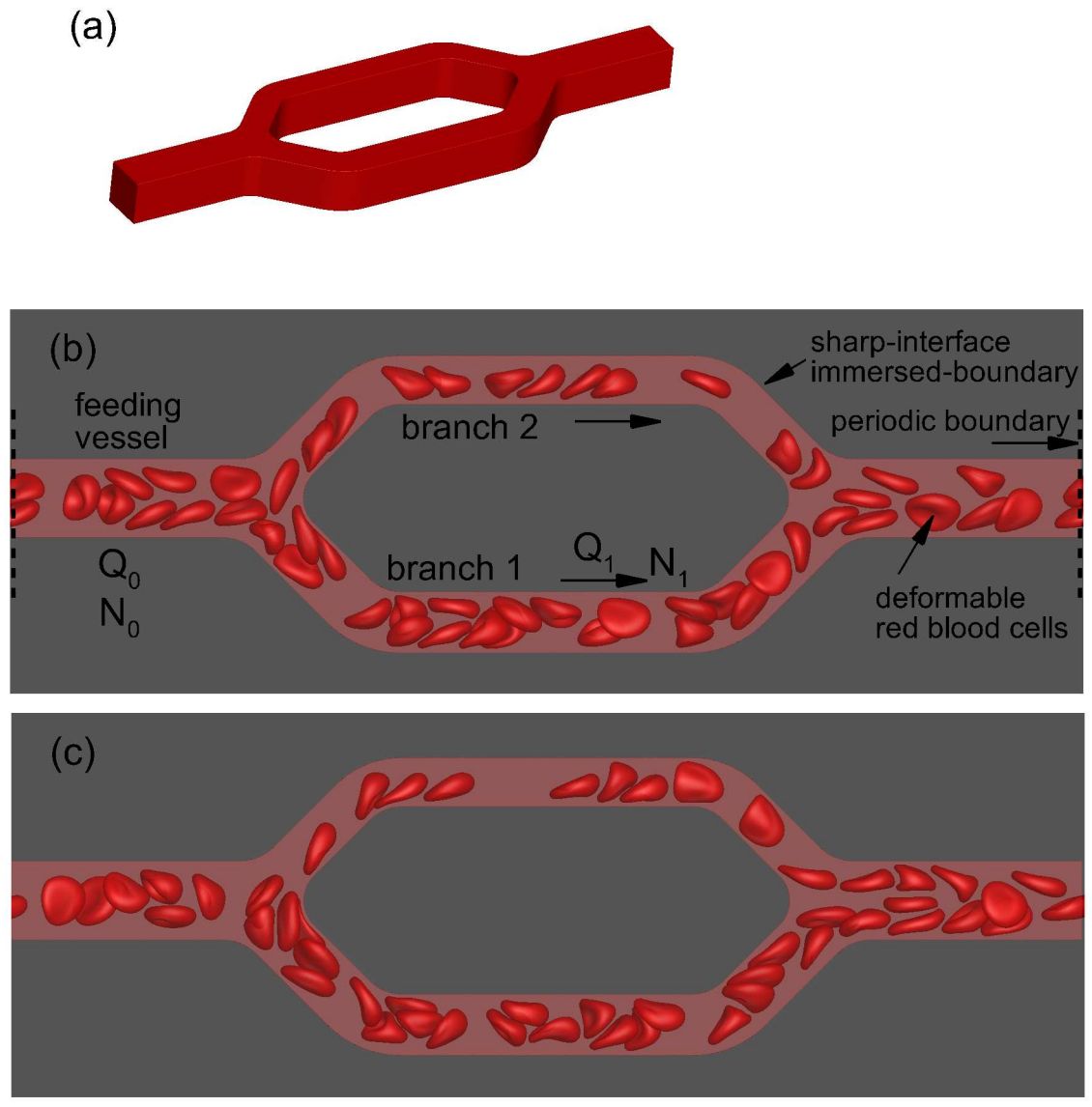

Figure 9: Validation study for multiple deformable interfaces in presence of rigid boundaries: Flow of deformable RBC in a bifurcating and merging channel. (a) Geometry of the channel modeled in the simulation. (b)-(c) Snapshots from one simulation showing the flow of deformed RBCs through the channels. The arrows indicate the flow directions. The feeding vessel (left) bifurcates in to two daughter vessels of unequal cross-sections. $Q_{0}$ and $Q_{1}$ are the flow rates and $N_{0}$ and $N_{1}$ are the RBC number densities, in the feeding vessel and in branch 1, respectively. Note that the left and right boundaries are periodic. 
The geometry considered for our simulations is shown in figure 9 (a). We model a microfluidic channel of rectangular cross-section that bifurcates into two daughter branches of unequal cross-sections. After a certain distance, the daughter branches again merge to form one channel. The cross-sections of the feeding branch (Branch 0) and one daughter branch (Branch 1) are fixed at $12 \times 12 \mu \mathrm{m}^{2}$ and $12 \times 9.3 \mu \mathrm{m}^{2}$, respectively, while that of $\mathrm{R} 1 \mathrm{C} 10$ Branch 2 is varied from $12 \times 3.7 \mu \mathrm{m}^{2}$ to $12 \times 7.5 \mu \mathrm{m}^{2}$. Thus, the flow rates in the daughter branches are varied by adjusting the cross-sectional area of Branch 2. The entire length of the device is $158 \mu \mathrm{m}$. The microfluidic geometry is modeled as a sharp-interface immersed boundary object, with its surface defined using a triangular mesh that is comprised of about 1.7 million triangles. The computational domain is $158 \times 49 \times 14 \mu \mathrm{m}^{3}$ which is discretized by $580 \times 180 \times 51$ Eulerian points. Each RBC surface is discretized by 5,120 Delauney triangles, and the number of RBCs considered for this problem is about 50. The flow is driven by a pressure differential between the inlet and outlet. Periodic boundary conditions are assumed for the velocity.

Snapshots from one simulation are shown in figures 9(b)-(c). As the cells reach the bifurcation they split into two daughter branches. Deformation of the RBCs is evident in the figures where many of them assume the so-called slipper shape and parachute shape as observed in experiments. From the simulations we obtain the ratio of the flow rates $Q_{1} / Q_{0}$, and the ratio of the RBC number densities $N_{1} / N_{0}$, where $Q_{0}$ and $Q_{1}$ are the flow rates in the feeding vessel and Branch 1 , and $N_{0}$ and $N_{1}$ are the RBC number densities. Generally, the flow rates and number densities vary with time, so time averages are done in computing the ratios once the flow has reached a quasi-steady state. Figure 10 presents $N_{1} / N_{0}$ as a function of $Q_{1} / Q_{0}$. If the cells were to distribute in proportion to the flow rates, the plot of $N_{1} / N_{0}$ versus $Q_{1} / Q_{0}$ would be a straight line with a slope of unity. Instead, a nonlinear variation in the form of a half-sigmoidal curve is noted. Such a sigmoidal curve is the result of a disproportionate splitting of the $\mathrm{RBC}$ suspension at the bifurcation, and hence, of the Z-F effect. At $Q_{1} / Q_{0} \approx 0.85, N_{1} / N_{0}$ becomes one, i.e., all RBCs are flowing 


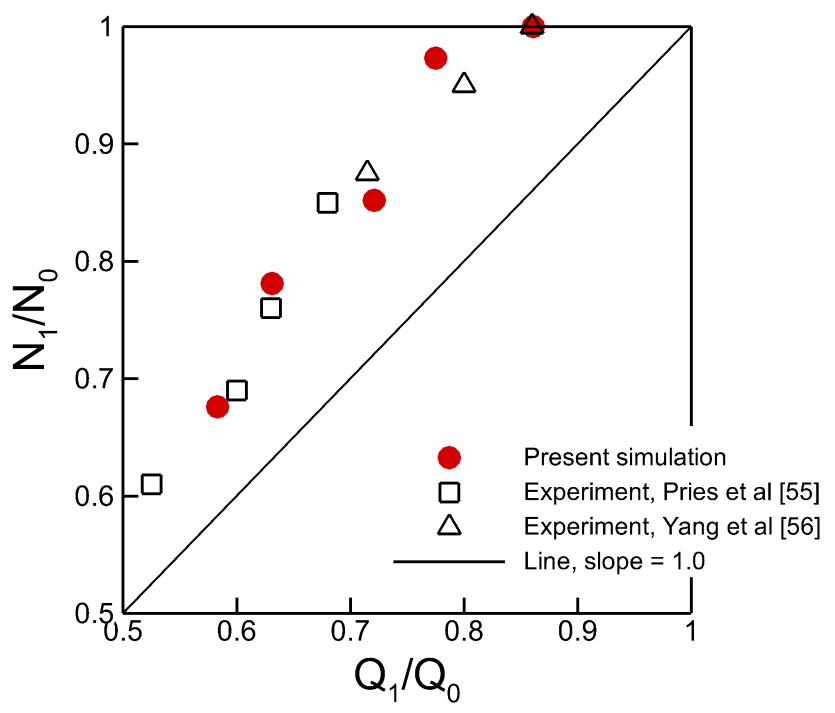

Figure 10: Multiple RBCs flowing in a bifurcating channel. Comparison of the present numerical results $(\bullet)$ with previous experimental measurements: in vivo data from Pries et al [55], and microfluidic measurements of Yang et al [56]. $N_{1} / N_{0}$ versus $Q_{1} / Q_{0}$ shows a half-sigmoidal variation and represents the Zweifach-Fung effect. 

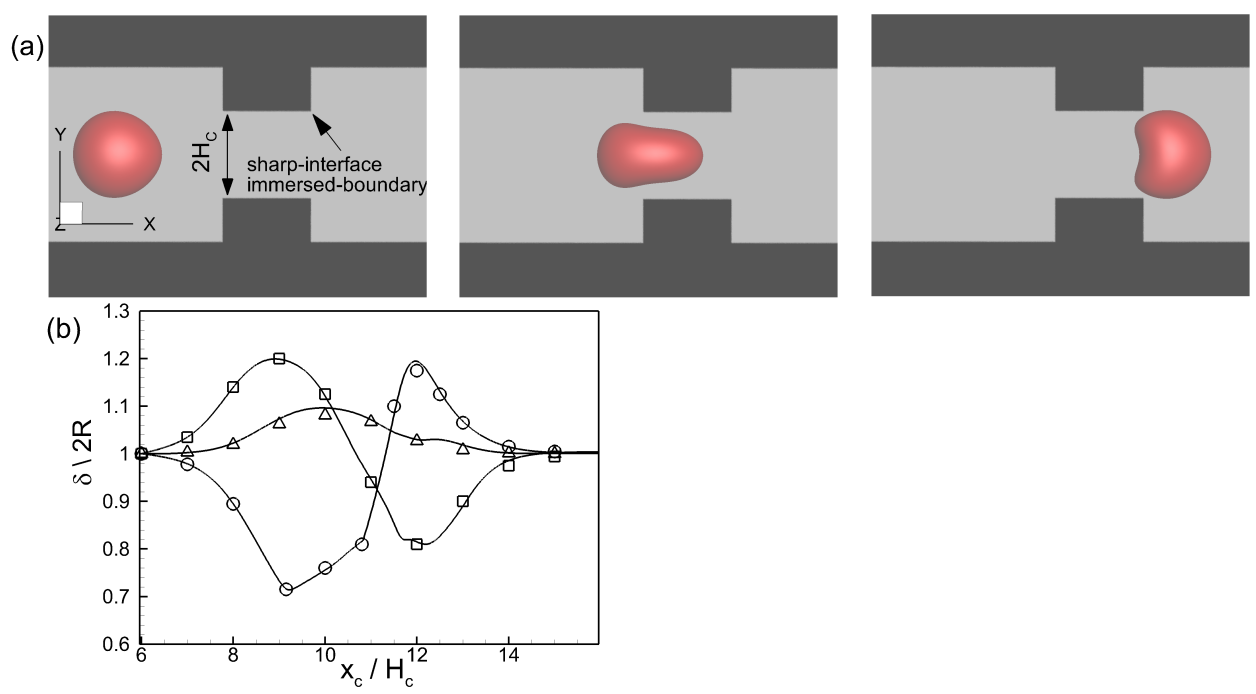

Figure 11: Validation with numerical results: Initially spherical cell moving through a microchannel constriction. (a) shows snapshots from our simulations at three time instances. (b) compares the capsule lengths in three directions obtained using the present methodology (-) and a spectral boundary integral method [17] ( $\square x, \circ y, \Delta z)$. Here $X_{C}$ is the instantaneous location of the cell center, and $R$ is the initial radius.

in Branch 1 while only plasma flows in Branch 2. This is the so-called plasma-skimming phenomenon also observed in vivo. Therefore, our numerical methodology is able to predict the plasma-skimming and Z-F effects.

In figure 10, the data from two experimental studies are also presented in addition to our numerical results: one is the in vivo measurement by Pries et al who considered blood flow through arteriolar bifurcations in the rat mesentery [55], and the other is the microfluidic measurements by Yang et al [56] using rigid spheres. The blood vessel diameters considered in the in vivo measurements, and the sphere to channel size in the microfluidic experiments were in the same range as considered here. The results from each of these experiments provide an example of a typical manifestation of the Z-F effect in the form of the sigmoidal variation of $N_{1} / N_{0}$ versus $Q_{1} / Q_{0}$. The results obtained from our simulation clearly capture this sigmoidal relation. 


\section{E. Comparison with a numerical study}

In the previous sections we validated the methodology against analytical results and experimental studies. In this section we validate our methodology against a previous numerical study. Furthermore, the specific example considered here validates the methodology in the presence of sharp rigid corners. [17] used a spectral boundary integral method to study the deformation of a capsule moving through a constricted microchannel. We recreate the conditions of this previous work, and compare with the results attained using our methodology. A microchannel of square cross-section with a rectangular constriction of height and length $2 H_{c}$ is considered as shown in figure 11. The microchannel geometry is modeled using the sharp-interface immersed boundary method, with the surface defined using a triangular mesh of about 1.5 million elements. This geometry is immersed within a bounding computational domain $20 H_{c} \times 4.5 H_{c} \times 5 H_{c}$. The Eulerian resolutions used are $400 \times 90 \times 100$ and $800 \times 180 \times 200$. An initially spherical cell is considered, with Skalak et al.'s model (Eq. 1). The cell surface is discretized using 5,240 or 20,480 triangles. The capillary number, R2C9 defined as $\mathrm{Ca}=\mu_{p} U_{c} / G_{s}$, was 0.1 , where $U_{c}$ is the average velocity in the unconstricted part of the channel in absence of the cell. Three values of the ratio of the fluid viscosity internal and external to the cell, $\mu_{c} / \mu_{p}$, are considered as $0.1,1$, and 5 . The ratio of the initial cell radius to the half-width of the constriction, $R / H_{c}$ is varied as $0.6,0.9$ and 1 .

Snapshots from a simulation $\left(R / H_{c}=1, \mathrm{Ca}=0.1, \mu_{c} / \mu_{p}=1\right)$ are presented in figure 11(a) which shows the squeezing of the cell through the constriction. Quantitative comparison with [17] is presented in figure $11(\mathrm{~b})$ for a case of $R / H_{c}=1, \mathrm{Ca}=0.1, \mu_{c} / \mu_{p}=0.1$ where deformed lengths of the cell in three directions are plotted as it squeezes through the constriction. Good agreement is observed between the results achieved using the different numerical methodologies for the specific case shown in the figure, as well as for varying parameters. Differences observed with different Eulerian and Lagrangian resolutions were negligible. The Eulerian resolution used for this case is slightly higher than that used with 
other cases, on account of the sharp corners of the constriction geometry. Thus the limiting factor with regard to mesh resolution was the sharp-interface geometry for this case, not the deformable cell.

\section{F. Additional considerations}

With the current methodology, a uniform rectangular Eulerian mesh is used to discretize the flow domain. As such, a natural consequence of arbitrary rigid boundaries immersed into the domain is that the degree of boundary alignment with the mesh can vary. The impact this may have on the results has been tested, considering two different notions of alignment.

The first notion of alignment is in the sense of symmetry of interfaces with respect to a natural centerline. For example, the case studied in Section III.E considered a centrally placed deformable cell flowing through a channel with a symmetric constriction. Ana-

lytically both the velocity field and evolution of the cell shape as it passes through the constriction will be symmetric about the centerline. Numerically speaking, with a symmetric shape like the constriction geometry it can be immersed into the domain such that the location of the Eulerian mesh points relative to the interface will be symmetric relative to the channel centerline. It can also be immersed in such a way that these relative locations are asymmetric. Cases for both of these configurations have been tested, and both the resulting velocity field and the shape evolution of the cell passing through the constriction were compared. For the fluid velocity fields, differences were observed to be less than $0.1 \%$. It is noted that in comparing the two cases, Eulerian fluid mesh points are not located at the same physical points in space, so an interpolation was used when comparing the results. This $<0.1 \%$ difference could therefore be attributed to the error in the interpolation used for comparison. In comparing the shape of the deformable cell, a negligible difference was 
also observed. We do note that as the Eulerian mesh resolution becomes more coarse, these differences may be more pronounced. However in all of our simulations we use fine enough resolutions to ensure relevant aspects of the flow are adequately resolved.

The second notion of alignment is in the sense that for rectangular rigid geometries, such as that considered in Section III.E, the rectangular Eulerian mesh can nicely align with the rigid interface. The majority of the validation cases in Section III involve rigid interfaces with curvature, thus testing this notion of alignment. In performing these cases, however, the effect of the symmetry of alignment was also tested. For the stationary sphere case (Section III.A), for example, at coarser mesh resolutions the error increased, as noted in the section. The location of the maximum error, at the coarser resolutions, was observed to shift to one side when the location of the sphere relative to the Eulerian mesh was slightly shifted. While the location shifted, the differences in the magnitude of the error were insignificant. So at the coarser resolutions, differences were observed in the symmetry of the error location, not in the magnitudes. This same general theme was observed for the other cases as well. At the finer Eulerian mesh resolutions, such as that of Figure 5, insignificant differences were observed in both the location and the magnitude.

In selecting the appropriate resolutions, the first part involves selecting an Eulerian resolution such that the relevant features of the flow will be resolved. Since the Lagrangian R1C5 mesh size is on the same order as the Eulerian mesh, as the cells deform, the degree to which the surface deformation is resolved is on the same order as the degree to which the surrounding flow is resolved. Our validation cases show that we are able to capture the essential mechanisms needed to understand flows in microvascular networks and microfluidic devices: the mechanics of overall cell populations (case D), and extreme deformation of an individual cell (case C). For case C, we have demonstrated the differences in result using different Lagrangian resolutions. 
As an example, the cells in Figure 9 are highly deformed, and the scale of the membrane deformation is small. However, this does not necessarily imply that the Eulerian resolution does not resolve the flow structures associated with this. In transferring information between the membrane and surrounding fluid, the scale of resolution is of the same order for each of the meshes associated with this. So they are consistent with one another. In other words, the deformation resolved on the Lagrangian mesh is consistent with the flow structure resolved on the Eulerian mesh.

Another important issue is the cell volume and surface area preservation. This issue has been addressed in our previous works $[30,36,37,49]$. Specifically, the cell surface area and volume change over time were shown in [49] in the Supplemental Materials. The change in cell volume is within $0.1 \%$ as noted there. Note that we satisfy the incompressibility condition at every Eulerian node up to machine epsilon. The change in cell surface depends on the specific constitutive law. The Skakal et al's model (Eq. 1) is nearly area preserving. The area dilation in this model is controlled by the parameter $C$ in Eq. 1. For the RBCs, we keep the area change within $0.1 \%$.

\section{Demonstration of computational capability}

In this section we demonstrate the capability of our methodology to simulate multiple deformable and heterogeneous cells moving in highly complex geometries ranging from physiologically realistic microvascular networks to geometrically complex microfluidic devices. The focus here is to show that the methodology can reproduce some well known but complex microphysiological and biomicrofluidic phenomena. Each case has been selected to demonstrate specific aspects of the methodology, as outlined in Table II. Together these studies show the versatility of the methodology in addressing a diverse set of problems 


\begin{tabular}{|c|l|l|}
\hline Sub-Section & Description & Features demonstrated \\
\hline \hline A & $\begin{array}{l}\text { Flow of RBC in a mi- } \\
\text { crovascular network }\end{array}$ & $\begin{array}{l}\text { Ability to model physiologically realistic mi- } \\
\text { crovascular geometries with highly deformable } \\
\text { cells }\end{array}$ \\
\hline B & $\begin{array}{l}\text { Platelet and RBC inter- } \\
\text { action }\end{array}$ & $\begin{array}{l}\text { Ability to handle both rigid and deformable cells } \\
\text { flowing in a microvascular network }\end{array}$ \\
\hline C & Microfluidic cell sorting & $\begin{array}{l}\text { Versatility of the methodology going beyond mi- } \\
\text { crovascular flows; diverse cell types in microflu- } \\
\text { idic devices }\end{array}$ \\
\hline D & $\begin{array}{l}\text { Geometrically complex } \\
\text { and integrated lab-on- } \\
\text { chip devices }\end{array}$ & Highly complex microfluidic geometry \\
\hline
\end{tabular}

Table II: Specific objective of each of the demonstration studies considered

\section{A. Flow of RBC in microvascular network}

Our first demonstration is the flow of many deformable RBCs in a complex microvascular network as shown in figure 12. The vascular network is numerically generated as described in $\S I I-D .2$ and following an in vivo image of human cerebral microvasculature given in [39]. The network considered in our simulation is comprised of 23 vessels of circular crosssection, with multiple bifurcations, trifurcations, and mergers, while several vessels are highly winding. The diameter of the vessels ranges from 11 to $17 \mu \mathrm{m}$, and the length of the vascular segments between two branch points ranges from approximately 18 to $70 \mu \mathrm{m}$. The walls of the vessels are not deformable, and are treated as sharp-interface immersed boundaries with the entire surface represented by approximately 1.25 million triangular elements. The computational domain is $131 \times 109 \times 65 \mu \mathrm{m}^{3}$, and the Eulerian resolution used is $480 \times 400 \times 240$. Multiple inlet and outlet vessels are present: the inlets are at the right and top boundaries of the domain, and the outlets are at the left and bottom boundaries. The flow is driven by pressure differences at the inlets and outlets. Periodic boundary conditions are assumed for the velocity. Cells leaving the outlets are also put back at the inlets. Each cell surface is discretized by 5120 triangular elements, and at any 

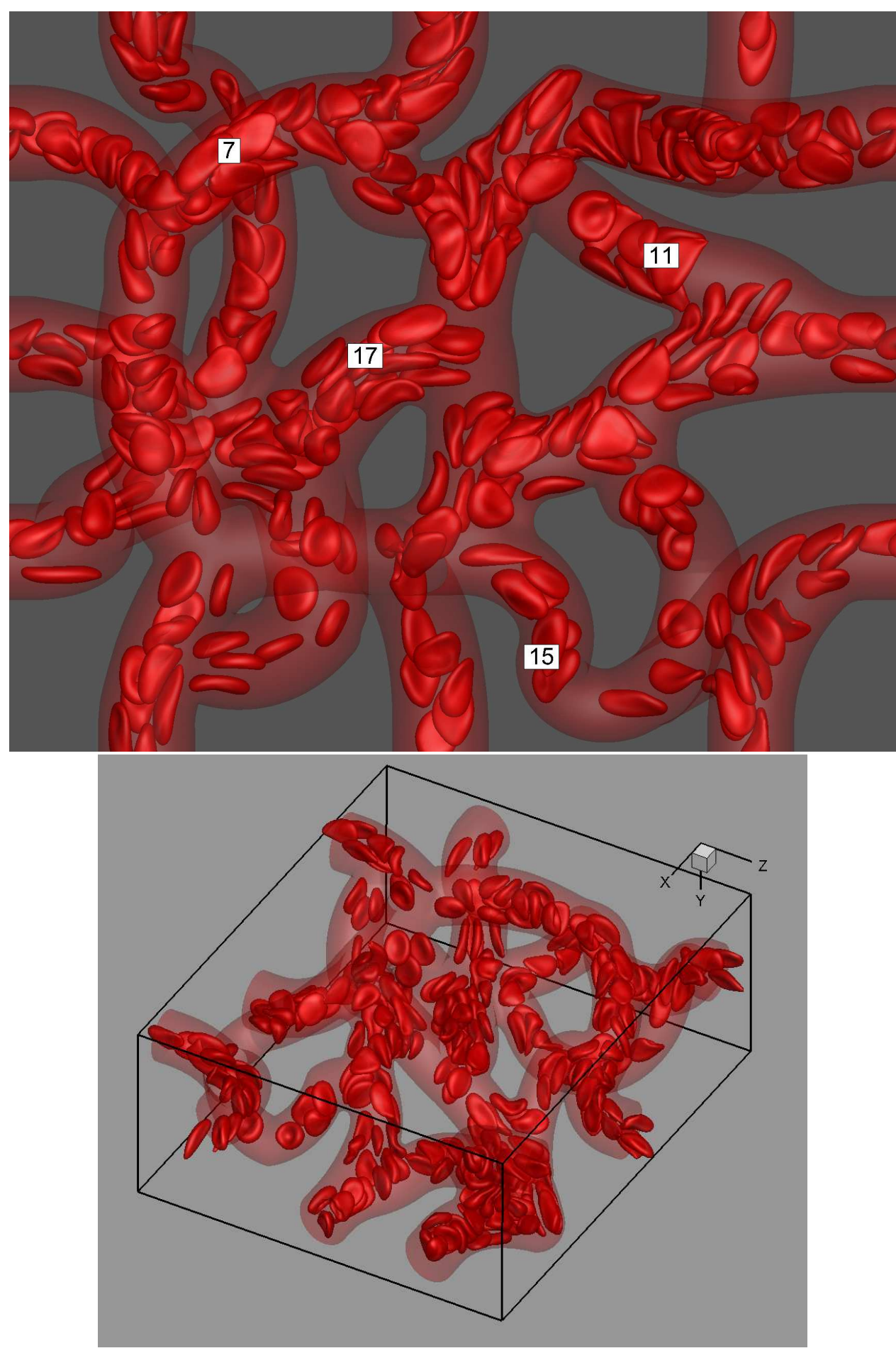

Figure 12: Demonstration of computational capability: Simulation of many deformable RBCs flowing in a microvascular network. Top figure is a view along the $y$ axis, and bottom figure is an isometric view. Some vessels are identified by numbers as needed for the discussion. 
instance the network contains approximately 300 RBCs.

A snapshot of the simulation at one time instance is shown in Figure 12. Extreme deformation of the cells is evident in this figure. Also noted are the parachute and slipper shapes which are the well-known characteristic shapes of RBCs flowing in capillary vessels. The simulation is conducted over a dimensionless time of $\gtrsim 800$, and the cell distribution in different branches evolves naturally. As can be seen in the figure, distribution of blood cells is highly nonhomogeneous across the network: some branches are filled with cells while others have only a few cells. For example, the hematocrit in Vessel 15 is $12 \%$ while that in Vessel 7 is $30 \%$. This inhomogeneity in cell distribution is a hallmark of blood flow in microvasculature. Also, in several branches the cell distribution over a cross-section of the vessels is not symmetric. This asymmetric distribution is due to the plasma skimming effect at the bifurcations which we predicted here and also earlier in $§ I I I-D$. Such asymmetric cell distribution is another hallmark of microvascular blood flow that the current simulation is able to predict.

To the best of our knowledge, this is the first 3D simulation of blood flow in a microvascular network that simultaneously resolves the large deformation of every blood cell while taking into account the complexity of the network architecture.

The simulation platform has the potential to be used as a virtual framework to understand many microcirculatory processes with cellular scale details in physiologically realistic vascular architecture. As an illustration, we extract the time-averaged velocity contours over the cross-sections of certain branches as shown in figure 13. A wide variability in the average velocity is observed among different vessels in the network. In certain vessels, e.g., in Branch 5 and 24, a nearly axisymmetric but "blunt" (non-parabolic) velocity profile is observed, which is a well-known characteristic of blood flow in small vessels. In 


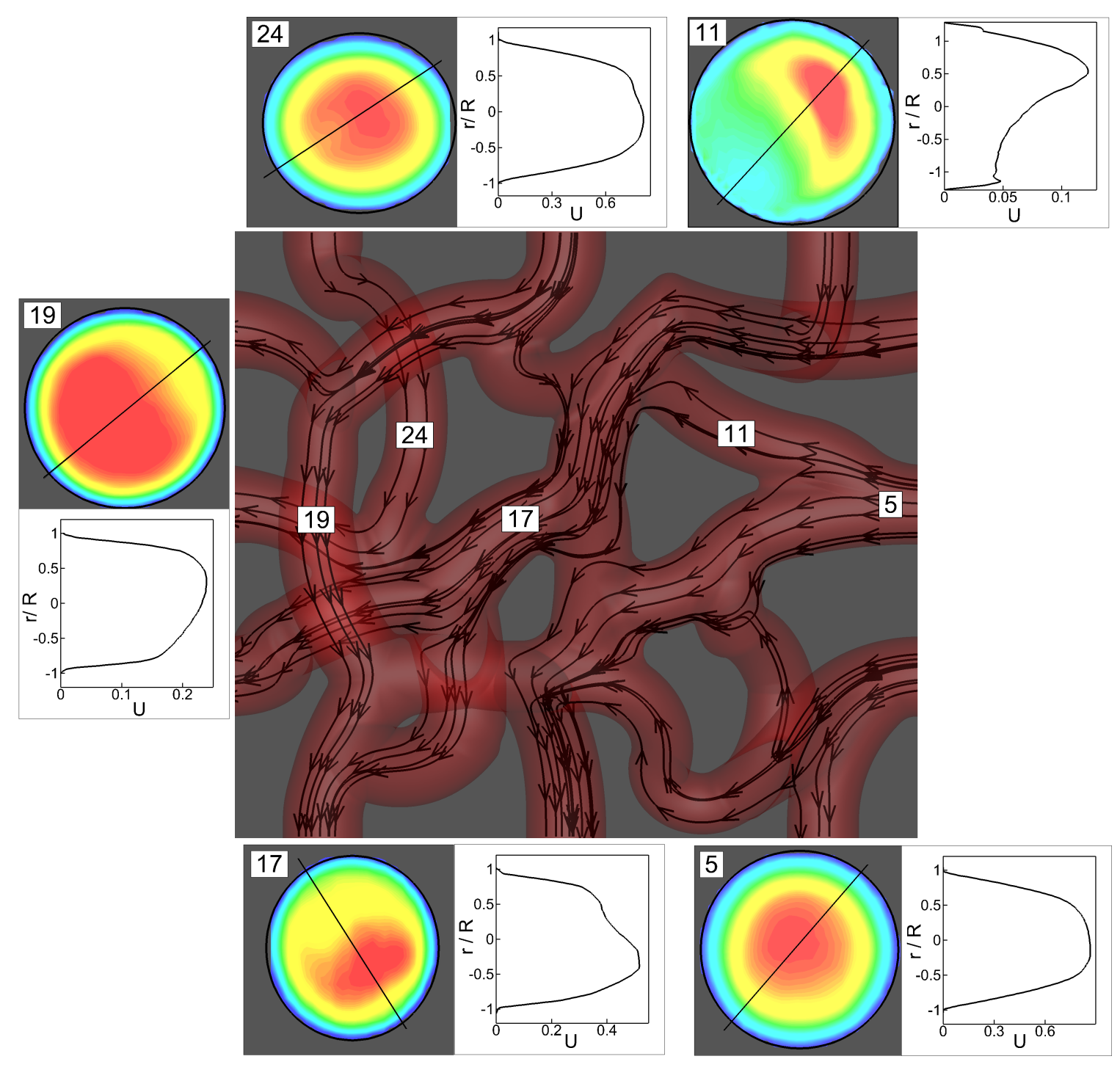

Figure 13: Streamlines, and time-averaged velocity contours at vessel cross sections in certain selected vessels for the microvascular network simulation. Also shown are the average velocity profiles across radial directions as indicated by the black lines. Well known 'blunt' velocity profiles, as well as radially non-axisymmetric profiles are noted. 
contrast, however, the velocity profiles in several other vessels, for example Branch 11 and 17, are highly non-axisymmetric. Such non-axisymmetric velocity profiles are observed in post-bifurcation daughter branches. The non-axisymmetry is also observed in the cell-free region along the vascular walls. These are due to the RBCs flowing along one side of a vessel after they split at the bifurcation, and have been noted in prior experiments (see, e.g. $[4,55,57])$.

As mentioned previously, most computational studies of cellular-scale blood flow to date have considered flow in unbranched, straight vessels in which the average velocity profile and cell-free region appear to be axisymmetric. In contrast, the present simulations show that these hemodynamic quantities are non-axisymmetric in many vessels depending on the architecture of the network as observed in vivo [57]. Such non-axisymmetric profiles may have a significant effect on wall shear stress and, hence, on endothelial cell response, as well as deposition of drug and other particulates. The present methodology can be used to address these critical microhemodynamic issues.

\section{B. Platelet and RBC interaction in microvascular network}

This section demonstrates the capability of the simulation methodology to deal with multiple deformable cells and moving rigid particles in a complex microvascular network. The specific example is the flow and interaction of RBCs and platelets in a network. As noted before, RBCs are highly deformable, while inactivated platelets behave as rigid particles. Normal platelets are of an oblate spheroidal shape with long axis $\sim 3.6 \mu \mathrm{m}$ and short axis $\sim 1 \mu \mathrm{m}$. We model the platelets as moving rigid objects using the GNIBM as described

in $\S I I-D .2$. Each platelet surface is represented by the analytical equation for a spheroid, and discretized using a surface mesh of 1,280 triangular elements for the force and torque computations. Each RBC surface is discretized by 5,120 triangular elements. The compu- 
tational domain is taken to be $65 \times 59 \times 33 \mu \mathrm{m}^{3}$, and is discretized using $240 \times 216 \times 120$ Eulerian points. The vascular network is immersed in the domain. Vessel surfaces are treated using the GNIBM and discretized by approximately 325,000 triangular elements.

A snapshot from our simulation is shown in figure 14(a). Because of their deformation, RBCs flowing in a vessel experience a hydrodynamic lift and migrate laterally towards the center of the vessel. The interaction between the RBCs and platelets causes the latter to marginate towards the wall. Platelet margination is the first step in the formation of a blood clot. Rigorous hydrodynamic analyses of platelet margination and the role of RBCs is emerging only recently, and has been limited to simple linear flows and in straight tubes. It is unknown how vascular bifurcations and mergers influence platelet margination. The representative simulation shown in figure 14 would allow such an analysis to be performed. As an illustration, we have extracted platelet trajectories from the simulation, and plotted the distance between the platelets and the vascular wall over time in figure 14(b). The platelets considered in this figure were released near the center of a vessel. It is observed that the wall-to-platelet distance decreases over time as the platelets marginate towards the wall. Therefore, the present methodology can successfully predict RBC-induced margination of platelets in a microvascular network. The methodology can be readily extended to study other similar problems of immense physiological significance, such as margination of white blood cells and circulating tumor cells, as well as RBC-induced drug deposition in realistic vascular geometry.

\section{Microfluidic cell sorting}

In the previous two demonstrations, we have considered physiological scenarios with internal viscous flows. The next demonstration deals with a microfluidic device involving external flows and includes deformable interfaces, moving rigid bodies, and non-moving boundaries. 


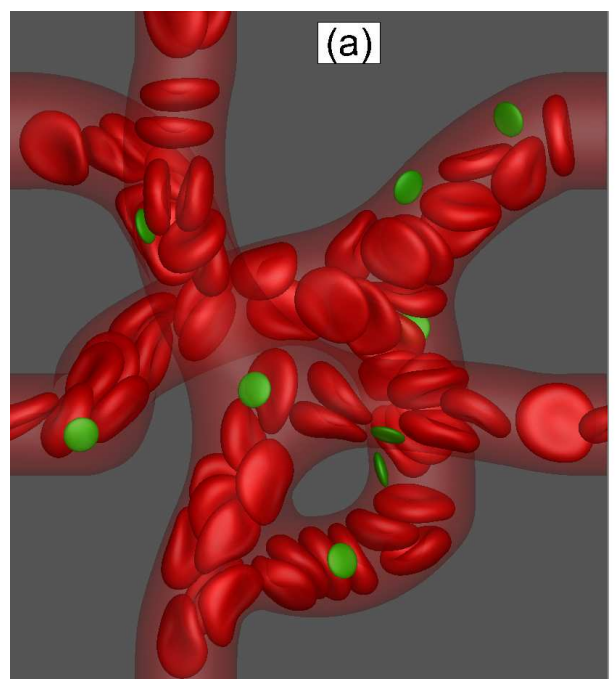

(b)

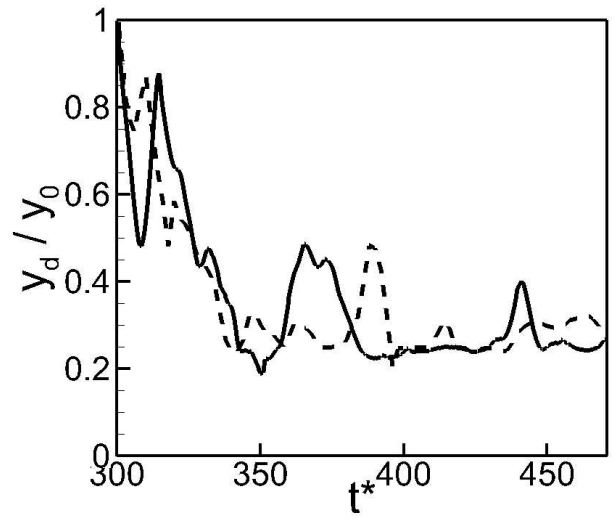

Figure 14: Platelet and RBC interaction in a microvascular network. (a) A snapshot from the simulation is shown. RBCs are shown in red, and platelets in green. (b) Platelet margination towards the vascular wall is shown by plotting the ratio of the platelet-to-wall distance $\left(y_{d}\right)$ to the initial distance $\left(y_{0}\right)$ for two platelets. Platelets were released near the center of the vessels. Over time they marginate towards the wall and remain flowing close to the wall as evident from the drop in $y_{d} / y_{0}$.

Specifically, we consider a microfluidic cell sorting technique. Cell sorting is a common microfluidic technique used to separate different types of biological cells, such as circulating tumor cells and blood cells. One such device is an array of rigid cylinders of micrometer size $[8,58]$. The basic idea behind such devices is to exploit different trajectories that flowing cells take based on their size and deformability. In this section we demonstrate the ability of the simulation tool to predict cell separation in such a device.

The geometry considered in our simulation is an array of 16 cylindrical posts with central axis perpendicular to the plane of the fluid motion (figure 15). The diameter of each post is $14 \mu \mathrm{m}$ and length $16 \mu \mathrm{m}$. The computational domain is taken to be approximately $98 \times 98 \times 16 \mu \mathrm{m}^{3}$, and the Eulerian resolution used is $360 \times 360 \times 60$. The domain is periodic in two directions and wall bounded at the ends of the cylinders. The flow is driven by a mean pressure gradient and is normal to the cylinder length. The rigid cylinders 
are modeled as sharp-interface immersed boundaries, with each surface represented by the analytical equation for a cylinder. The cell suspension contains three different types of cells: deformable RBCs, rigid platelets, and a third type of cell that is stiffer than an RBC and bigger in size (12 $\mu \mathrm{m}$ in diameter). The latter type is representative of both white blood cells (WBC) and circulating tumor cells (CTC). The resolution used for each cell is the same as that in the previous two examples.

A snapshot from one simulation is shown in figure 15(a). The cells move around the posts as they are driven by the flow, and large deformations can be observed in each of the RBCs. The streamlines and velocity magnitude at the central $x-z$ plane are shown in figure 15(b) in absence of the cells, and a symmetric flow structure around each cylinder is observed as is the case for a Stokes flow. The interaction of the cells with the posts affects their trajectories, which are monitored in the simulation. Figure 15(c) compares the trajectories of an $\mathrm{RBC}$, a platelet, and a WBC/CTC, with the same initial position. The direction of the mean fluid flow is also shown. It is readily seen that the deviation of a cell trajectory from the flow direction increases with increasing cell size. The platelets, being the smallest cells, nearly follow the mean fluid path, while the WBC/CTCs follow a distinctly different path. As can be seen, the trajectory of the WBC/CTC quickly separates from that of the RBC and platelet. Over a time of approximately 0.02 seconds, more than $100 \mu \mathrm{m}$ of separation between the $\mathrm{WBC} / \mathrm{CTC}$ and the $\mathrm{RBC}$ is observed. These results demonstrate that the simulation tool can be used to design microfluidic cell sorting devices that exploit the deformability and size/shape-dependent sorting of cells in suspension.

\section{Geometrically complex and integrated lab-on-chip devices}

Microfluidic lab-on-chip devices often integrate many geometrically complex features in order to perform a complete analysis of a biological cell suspension $[8,59]$. The final demon- 


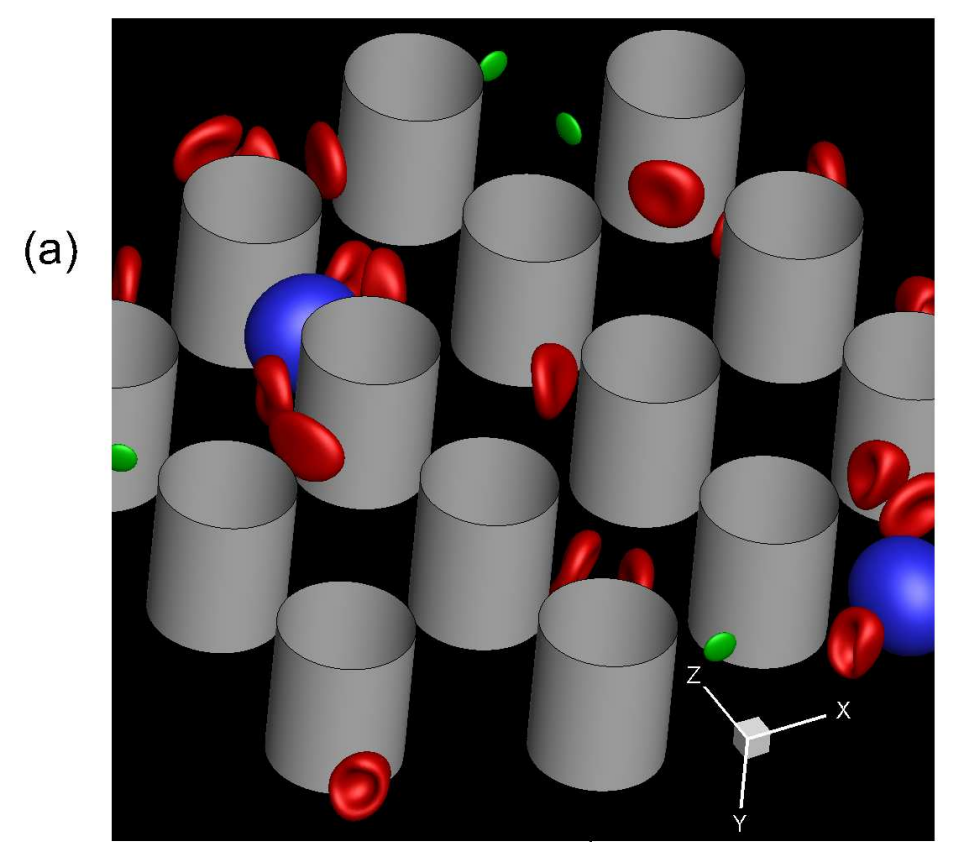

(b)

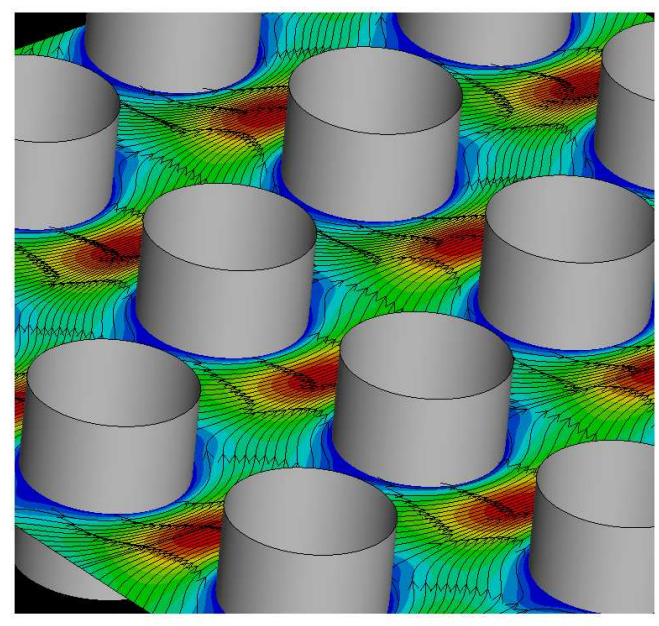

(c)

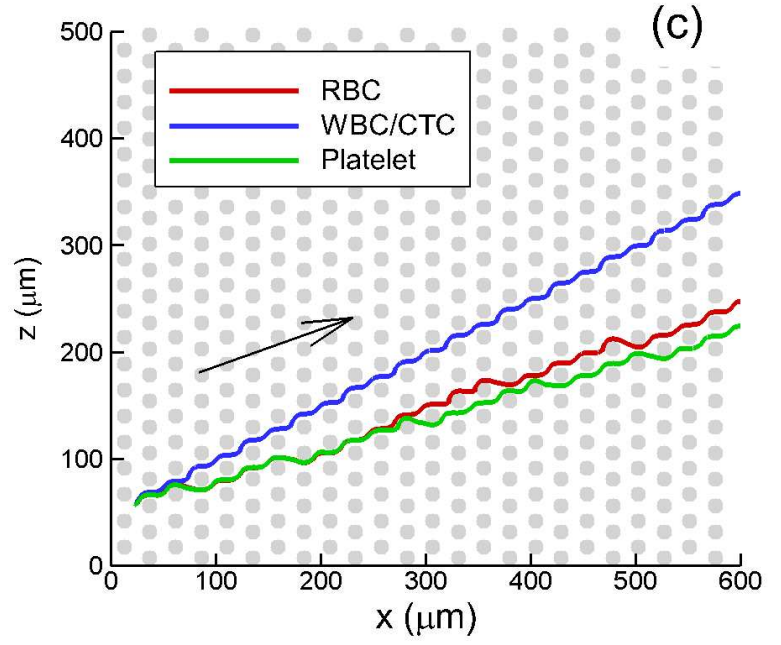

Figure 15: Simulation of cell sorting: flow of a suspension of heterogeneous cells through an array of cylindrical posts. (a) A snapshot from the simulation. Three types of cells are considered: deformable RBC (red), white blood cells (WBC) and circulating tumor cells (CTC) (blue), and rigid platelets (green). Flow is along the $x-z$ plane. (b) Streamlines and velocity magnitude at the central $x$ - $z$ plane. Velocity magnitude range in arbitrary dimensionless units is 0 (blue) to 0.6 (red). (c) Trajectories of an RBC (-), a WBC/CTC $(-)$, and a platelet $(-)$. The arrow indicates the mean flow direction. 
stration of our numerical methodology is in resolving deformable cell motion in such a complex device. A lab-on-chip device is modeled as depicted in figure 16(a). The device combines various geometrical features commonly utilized, such as a winding passage, sudden expansion, obstacles of different shapes, and channel bifurcations. The length of the device is approximately $250 \mu \mathrm{m}$, and it is immersed in a $250 \times 100 \times 10 \mu \mathrm{m}^{3}$ computational domain that is discretized using $900 \times 390 \times 35$ Eulerian points. The walls of the device are modeled as sharp-interface immersed boundaries and discretized by approximately 2.1 million triangular elements. Flow is driven by an applied pressure difference at the inlet and outlets. Two different types of deformable cells, namely RBCs and WBC/CTCs, are injected at the inlet. Figure 16(b) shows a snapshot from the simulation with the distribution of highly deformable RBCs and relatively stiff WBC/CTCs. Large deformation of the cells can also be noted here as they flow through the winding passages and around the obstacles. Also shown is the trajectory of each cell which can be analyzed further to assess cell separation efficiency for the present device or similar devices. This example further demonstrates the capability of the present methodology to resolve flow of multiple heterogeneous and deformable cells in highly complex geometry such as that often encountered in integrated lab-on-chip devices. Another feature of this example is that it demonstrates the capability of the methodology to simulate flows involving boundaries with discontinuities in the surface curvature. Specifically this simulation involves an internal flow through a geometry with sharp corners, in addition to an external flow around objects such as the rectangular and triangular obstructions. The ability to simulate flows around sharp-edged obstructions such as these further highlights the versatility of the methodology with regard to boundary complexity. 

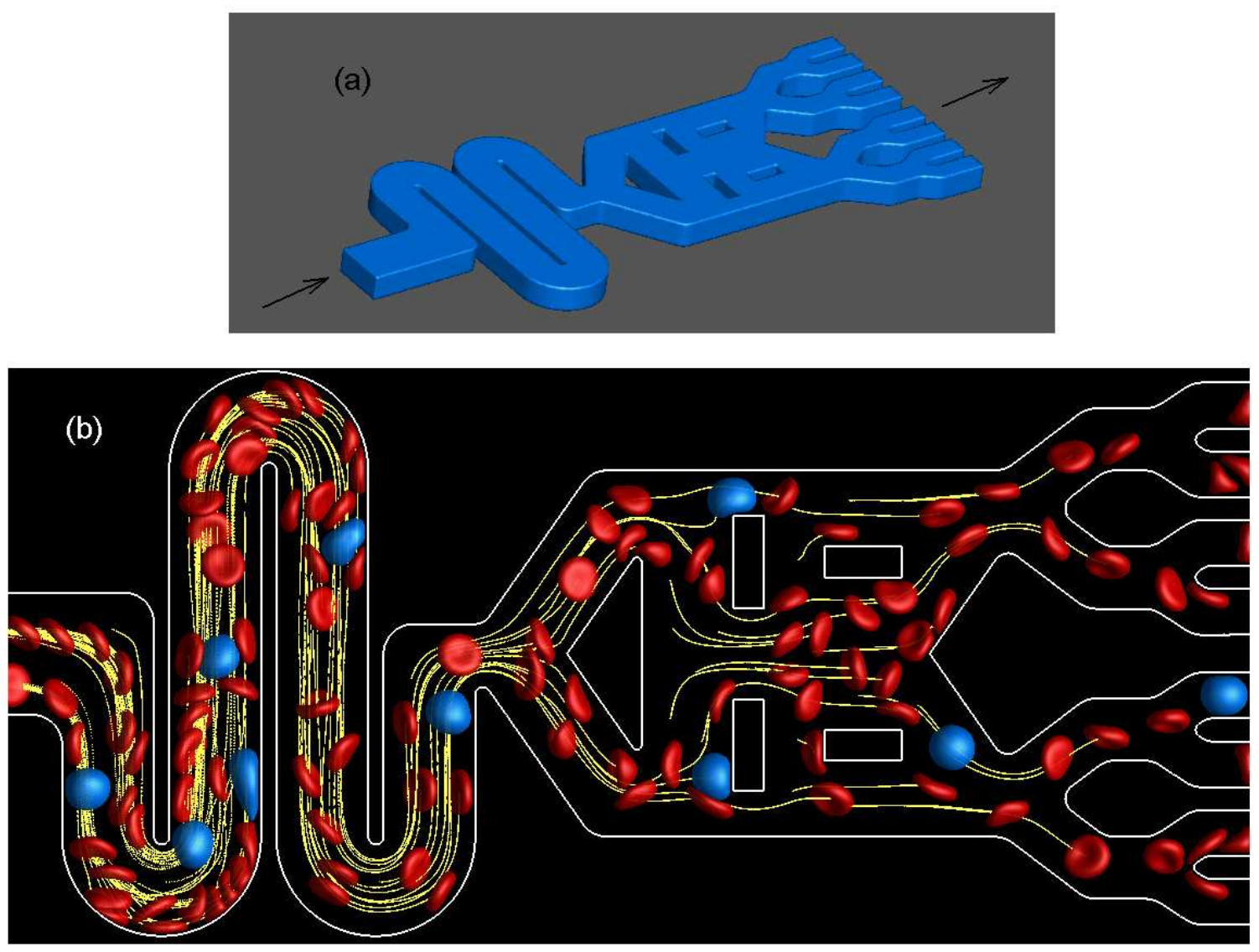

Figure 16: Simulation of the flow of a suspension of deformable RBCs and WBC/CTCs in a geometrically complex lab-on-chip device. (a) 3D view of the device modeled. The mean flow is from left to right. The device consists of winding channels, a sudden expansion, obstacles of different shapes, and bifurcations. (b) A snapshot from the simulation. RBCs are shown in red, and WBC/CTCs are in blue. Sharp-interface boundaries are in white. Trajectories of a few cells are shown in yellow. 


\section{Discussion}

$\mathrm{R} 2 \mathrm{C} 2,3$

The scientific objective of the present work was to develop a novel numerical technique for simulating the flow of deformable cells and rigid bodies in arbitrarily complex geometries as encountered in diverse types of scenarios ranging from microvascular networks to microfluidic devices. To meet this objective, we have demonstrated the versatility of the $\mathrm{R} 2 \mathrm{C} 1$ methodology in dealing with diverse types of interfaces and geometries by considering a large number of validation and demonstration studies. The extensive validations and demonstrations presented here underscore the strength and uniqueness of the computational tool developed.

The present methodology provides a novel means of simulating both deformable and rigid cells flowing within arbitrarily complex geometries. It seamlessly integrates different modeling components dealing with rigid non-moving boundaries, rigid moving boundaries, and highly deformable interfaces governed by complex nonlinear elasticity. In addition, multiple fluids with different viscosity, can be considered. A finite-element method is used to deal with the deforming interfaces, and the coupling between the fluid flow and interface deformation is obtained via the continuous-forcing IBM. A sharp-interface ghost-node IBM is used to treat the vascular walls and moving rigid bodies. A novel technique to couple the rigid body dynamics with the fluid flow is also presented.

A series of validation studies is presented to establish the accuracy of the methodology against analytical theory, experimental data, and previous numerical results. The overall goal of these is to systematically test the ability of the methodology to accurately capture the wide range of phenomena commonly encountered in the complex biological flows considered. As such, each case is studied with a specific purpose in mind, as summarized in Table I. The accuracy of the rigid interface methodology is first verified by modeling a rigid 
stationary sphere in a shear flow. This case is also used to establish a general mesh resolution criteria for rigid interfaces. The accuracy of the moving rigid interface methodology is then established. Then we establish the accuracy for moving and non-moving rigid boundaries in close proximity. The accuracy of the methodology for simulations involving both rigid and deformable interfaces is then studied. We first model a red blood cell aspirated into a micropipette, and compare our results with experimental data. An excellent agreement between the two is observed, demonstrating the ability to resolve the close interaction of a rigid boundary with a deforming interface under extreme deformation of a cell. We then simulate multiple deformable RBCs flowing through a bifurcating channel. We show a good agreement with experimental data thus demonstrating the ability of the methodology for high hematocrit flows. Lastly we recreate a simulation previously performed using an alternate numerical method in which a capsule flows through a rectangular constriction. It is shown that we are able to capture the transient cell dynamics with the same degree of accuracy.

The method has been shown to be highly versatile, and able to model very complex geometries, as evident from the extensive list of demonstrations. Each demonstration case has specific objectives that serve to highlight different features of the methodology, as outlined in Table II. We have simulated RBCs flowing in a microvascular network as a demonstration of the ability to model physiologically realistic microvascular networks with highly deformable cells. We have simulated RBCs and platelets interacting in microvascular network flow, demonstrating the ability to handle both rigid and deformable cells flowing in such complex geometries. Going beyond microvascular flows, we have demonstrated the ability of the methodology to be extended to complex microfluidic devices handling diverse types of cells. These demonstrations collectively establish the versatility of the numerical tool to address a diverse range of problems.

With each of these demonstrations, it is shown that the methodology can predict several 
complex microhemodynamic phenomena observed in vascular networks and microfluidic devices, such as the plasma-skimming and Zweifach-Fung effects, platelet margination, and deformability- and size-based cell separation. It is noted that for each case it is possible to conduct an extensive hemodynamic study, however the present purpose is to demonstrate the wide range of potential the present methodology offers.

As the validation and demonstration cases show, a major advantage of the current methodology is that it is not limited to any specific geometry; as such, vessels with multiple bifurcations and mergers, as well as tri- and multi-furcations, can be easily modeled. The ability to model fully three-dimensional cellular-scale blood flow in such arbitrary and highly complex geometry while simultaneously resolving the details of the deformation and dynamics of every cell represents the forefront of the current state-of-the-art. The present methodology is robust and versatile, and has the potential to scale up to extremely large microvascular networks at organ levels. To the best of our knowledge, the specific demonstration of many deformable blood cells flowing through the physiologically realistic microvascular network that is presented here is the first of its kind.

While the approach we have taken is based on immersed boundary methods, there are various other approaches that may be considered for simulating the types of interfaces modeled here. In the context of finite-difference based methods, perhaps the most direct approach is to use a boundary-fitted computational mesh to discretize the flow domain. Benefits of this approach with regard to accuracy are straightforward. All boundaries align with the computational grid, and thus the accuracy of the method is simply in accordance with the discretization scheme. Modeling arbitrarily complex boundaries, however, can significantly hinder the performance and limit the overall robustness, especially when considering moving complex boundaries. The benefits of using IBMs, or others, in lieu of this approach have been well documented, and owe mainly to the significant increase in computational efficiency outweighing decreases in accuracy, if any. 
Common alternatives to IBMs for modeling interfaces such as those considered here are boundary integral methods, stochastic particle methods, and lattice-Boltzmann methods. A detailed review on each of these methods for modeling blood flow was performed in [1], and the salient features on each have been used here in the subsequent discussions. Boundary integral methods have been shown to be highly efficient and accurate in simulating multiphase flows, although they are strictly limited to linear flows and can present difficulties with regard to specifying boundary conditions. While the level of accuracy achieved using these approaches is typically higher than that of IBMs, it is unclear if a method of this type can be efficiently extended to model the large-scale highly complex three-dimensional geometries considered here. Most studies using boundary integral methods are limited to simple geometries.

Dissipative particle dynamics (DPD) provides the most common basis for stochastic particle methods. It has been used to simulate blood cells within various types of geometries $[14,22,23]$, and has been shown to be highly efficient. However, the means by which noslip conditions are achieved at solid boundaries requires attention with DPD, as particle repulsions generally cannot achieve this alone. Thus extra measure must be taken in order to accurately impose no-slip conditions. The order of accuracy of this method for commonly used Verlet-type integrators tends to be first-order [67], while generally speaking the error order is unclear on account of the random and dissipative forces in the integration algorithm [68]. In addition, both the accuracy of the flow solver and the smoothness of resolution of the deformable cell interfaces are of a lower order than with the IBM approach. Also, most studies in the literature offer little information on the implementation of multiple fluids of different viscosity with this or other particle-based methods. In contrast, implementing multiple fluids of different viscosity is straightforward using a front-tracking method, as is evident from many works in the literature using this approach $[28,30,36,38,49]$. The degree of geometric complexity that can be achieved with DPD seems to be on the same order as with our IBM approach. 
Lattice-Boltzmann (LB) methods involve a particle-based, meso-scale approach and are well suited to modeling complex geometries, in addition to being efficiently parallelized. Simulation of complex boundaries using LB is typically done using either IBMs or other approaches, and are typically simulated to first-order accuracy. As the theoretical basis on which these methods are founded naturally results in compressible fluids being modeled, care must be taken in order to simulate incompressible flows approaching zero inertia. On the other hand, with a finite-volume based IBM approach such as ours, both zero and finite inertia flows can be simulated with ease.

Evidently, the methodology developed here offers an efficient, and often better, alternative to these other commonly used methods. It can be employed in a relatively straightforward manner, and has been shown in this study to be both versatile and accurate through the extensive validation and demonstration cases. It provides a viable means of bridging the gap between resolving the sharp features of extreme cell deformation and modeling flows through large-scale complex geometries.

\section{Conclusions}

We have presented a computational approach to modeling cellular-scale blood flow in complex geometry. Our approach is based on immersed boundary methods which allow modeling flows in arbitrarily complex geometry without requiring a body-conforming computational mesh. In addition to handling arbitrary geometry, the methodology resolves the deformation and dynamics of every blood cell with high fidelity. Specifically, the large deformation of red blood cells, motion of rigid platelets, and heterogeneous cellular suspensions are accurately resolved. Thus, the computational tool allows the simulation of 'whole' blood at the cellular-scale in physiologically realistic microvascular networks that are characterized 
by bifurcating, merging and winding vessels, as well as in geometrically complex lab-on-chip devices.

In the future, the computational tool can be used to address many unresolved issues related to microvascular blood flow. For example, it can be used to address the influence of vascular bifurcations and mergers on platelet accumulation, and hence, on the genesis of microthrombus. The tool can be used to compare blood flow patterns in normal and tumor microvasculature, and provide further insights on tumor microcirculation. It can be used to study the origin, characteristics and implications of self-sustained oscillations observed in microvascular blood flow [60]. Furthermore, the methodology can be used as a virtual tool to study the transport and deposition of submicron drug particulates in microvascular networks, and optimize their physical properties for organ- and tumor-specific delivery. It can also be used to design microfluidic lab-on-chip devices for efficient cell separation based on cell deformation, shape, and size.

\section{REFERENCES}

1. J.B. Freund, 'Numerical simulation of flowing blood cells'. Ann. Rev. Fluid Mech. 46:67-95 (2014).

2. Y. Imai, T. Omori, Y. Shimogonya, T. Yamaguchi, and T. Ishikawa. 'Numerical methods for simulating blood flow at macro, micro, and multi scales'. J. Biomech. (In press) (2015). doi:10.1016/j.jbiomech.2015.11.047.

3. R.M. Berne and M.N. Levy, Cardiovascular Physiology. Mosbey. 8th ed. (2000).

4. A.S. Popel and P.C. Johnson, 'Microcirculation and hemorheology'. Ann. Rev. Fluid Mech. 37:43-69 (2005).

5. Y.C. Fung, Biomechanics: Circulation, 2nd ed, 1997, Springer.

6. A.R. Pries, T.W. Secomb and P. Gaehtgens. 'Biophysical aspects of blood flow in the microvasculature'. Cardiovascular Res. 32:654-667 (1996).

7. A.L. Fogelson and K.B. Neeves. 'Fluid mechanics of blood clot formation'. Ann. Rev. Fluid Mech. 47:377-403 (2015).

8. J.M. Martel and M.Toner. 'Inertial focusing in microfluidics'. Annu. Rev. Biomed. Eng. 16:371-396 (2014).

9. D.B. Kolesky, R.L. Truby, A.S. Gladman, T.A. Busbee, K.A. Homan, and J.A. Lewis. '3D bioprinting of vascularized, heterogeneous cell-laden tissue constructs'. Advanced Materials 26:3124-3130 (2014). 
10. L.E. Bertassoni, M. Cecconi, V. Manoharan, M. Nikkhah, J. Hjortnaes, A.L. Cristino, G. Barabaschi, D. Demarchi, M.R. Dokmeci, Y. Yang, A. Khademhosseini. 'Hydrogel bioprinted microchannel networks for vascularization of tissue engineering constructs'. Lab. Chip 14:2202-2211 (2014).

11. J.O. Barber, J.P. Alberding, J.M. Restrepo, and T.W. Secomb. 'Simulated twodimensional red blood cell motion, deformation, and partitioning in microvessel bifurcations'. Ann Biomed Eng. 36:1690-1698 (2008).

12. H.C. Woolfenden and M.G. Blyth. 'Motion of a two-dimensional elastic capsule in a branching channel flow'. J. Fluid Mech. 669:3-31 (2011).

13. X. Yin, T. Thomas, J. Zhang. 'Multiple red blood cell flows through microvascular bifurcations: Cell free layer, cell trajectory, and hematocrit separation'. Microvascular Res. 89:47-56 (2013).

14. X. Li, A.S. Popel, and G.E. Karniadakis. 'Blood-plasma separation in Y-shaped bifurcating microfluidic channels: A dissipative particle dynamics simulation study'. Phys Biol. 9:1478-3975 (2012).

15. C. Sun and L.L. Munn. 'Lattice Boltzmann simulation of blood flow in digitized vessel networks'. Comput Math Appl. 55: 1594-1600 (2008).

16. A. Leyrat-Maurin and D. Barthes-Biesel. 'Motion of a deformable capsule through a hyperbolic constriction'. J. Fluid Mech., 279:135-163 (1994).

17. S. Park and P. Dimitrakopoulos. 'Transient dynamics of an elastic capsule in a microfluidic constriction'. Soft Matter, 9:8844-8855 (2013).

18. C. Rorai , A. Touchard, L. Zhu, L. Brandt. 'Motion of an elastic capsule in a constricted microchannel'. Eur. Phys. J. E. 38:49-62 (2015).

19. J. Freund. 'The flow of red blood cells through a narrow spleen-like slit'. Phys. Fluids, 25:110807 (2013).

20. L. Zhu and L. Brandt. 'The motion of a deforming capsule through a corner'. J. Fluid Mech. 770:374-397 (2015).

21. W. Wang, T. Diacovo, J. Chen, J. Freund, and M. King. 'Simulation of platelet, thrombus and erythrocyte hydrodynamic interactions in a 3D arteriole with in vivo comparison'. PLoS ONE, 8(10) e76949:1-11 (2013).

22. D. Fedosov, M. Peltomaki, and G. Gompper. Deformation and dynamics of red blood cells in flow through cylindrical microchannels. Soft Matter, 10:4258-4267 (2014).

23. Z Zhang, E. Henry, G. Gompper, and D. Fedosov. Behavior of rigid and deformable particles in deterministic lateral displacement devices with different post shapes. J. Chemical Phys. 143:243145 (2015).

24. K. Vahidkhah, N. Fatouraee. 'Numerical simulation of red blood cell behavior in a stenosed arteriole using the immersed boundary-lattice Boltzmann method'. Inter. J. Numer. Methods Biomed. Eng. 28:239-256 (2012).

25. T. Skorczewski, L.C. Erickson, and A.L. Fogelson. 'Platelet motion near a vessel wall or thrombus surface in two-dimensional whole blood simulations'. Biophys. J. 104:1764$1772(2013)$.

26. R. Mittal and G. Iaccarino. 'Immersed boundary methods'. Ann. Rev. Fluid Mech. 37:239-261 (2005).

27. C.S. Peskin. 'The immersed boundary method'. Acta Numerica. 11:479-517 (2002).

28. X. Li, and K. Sarkar. 'Front-tracking simulation of deformation and buckling 
instability of a liquid capsule enclosed by an elastic membrane'. J. Comput. Phys. 227:49985018 (2008).

29. Y. Sui, H.T. Low, Y.T. Chew, and P. Roy. 'Tank-treading, swinging, and tumbling of liquid-filled elastic capsules in shear flow'. Phys. Rev. E 77:016310 (2008).

30. S.K. Doddi, and P. Bagchi. 'Lateral migration of a capsule in a plane Poiseuille flow in a channel'. Intl. J. Multiphase Flow 34:966-986 (2008).

31. C.D. Eggleton, and A.S. Popel. 'Large deformation of red blood cell ghosts in a simple shear flow'. Phys. Fluids 10:1834-1845 (1998).

32. R. Skalak, A. Tozeren, P.R. Zarda, and S. Chien. 'Strain energy function of red blood cell membranes'. Biophys. J. 13:245-264 (1973).

33. J. Mills, L. Qie, M. Dao, C. Lim, and S. Suresh. 'Nonlinear elastic and viscoelastic deformation of the human red blood cell with optical tweezers.' Molecular and Cell Biology 1:169-180 (2004).

34. S. Shrivastava, and J. Tang. 'Large deformation finite element analysis of nonlinear viscoelastic membranes with reference to thermoforming'. J. Strain Anal. 28:31-51 (1993).

35. O.-Y. Zhong-Can, and W. Helfrich. 'Bending energy of vesicle membranes: general expressions for the first, second, and third variation of the shape energy and applications to spheres and cylinders'. Phys. Rev. A 39:5280-5288 (1989).

36. A. Yazdani, and P. Bagchi. 'Three-dimensional numerical simulation of vesicle dynamics using a front-tracking method'. Phys. Rev. E 85:056308 (2012).

37. A. Yazdani and P. Bagchi. 'Influence of membrane viscosity on capsule dynamics in shear flow. J. Fluid Mech. 718:569-595 (2013).

38. G. Tryggvason, B. Bunner, A. Esmaeeli, N. Al Rawahi, W. Tauber, J. Han, S. Nas, and Y. Jan. 'A front-tracking method for the computations of multiphase flow'. J. Comput. Phys. 169:708-759 (2001).

39. S. Lorthois and F. Cassot. 'Fractal analysis of vascular networks: Insights from morphogenesis'. J. Theoretical Biology. 262:614-633 (2010).

40. C. Geuzaine and J.-F. Remacle 'Gmsh: a three-dimensional finite element mesh generator with built-in pre- and post-processing facilities.' Inter. J. Numerical Methods Eng. 79:1309-1331 (2009).

41. R. Mittal, H. Dong, M. Bozkurttas, F. Najjar, A. Vargas, and A. von Loebbecke. 'A versatile sharp interface immersed boundary method for incompressible flows with complex boundaries' J. Comput. Phys. 227:4825-4852 (2008).

42. D. Brown, R. Cortez, and M. Minion. 'Accurate projection methods for the incompressible Navier-Stokes equations.' J. Comput. Phys. 168:464-499 (2001).

43. J. Kim and P. Moin. 'Application of a fractional step method to incompressible Navier-Stokes equations.' J. Comput. Phys. 59:308:323 (1985).

44. J. Bell, P. Colella, and H. Glaz. 'A second-order projection method for the incompressible Navier-Stokes equations.' J. Comput. Phys. 85:257-283 (1989).

45. H. Choi and P. Moin. 'Effects of the computational time step on numerical solutions of turbulent flow.' J. Comput. Phys. 113:1-4 (1994).

46. H. Baruh. 'Analytical Dynamics.' McGraw-Hill, 1999.

47. M.P. Allen and D.J. Tildesley. 'Computer simulation of liquids.' Oxford Univ. Press, 1991.

48. C. Pozrikidis. 'Introduction to theoretical and computational fluid dynamics.' Ox- 
ford Univ. Press, 2nd Ed., 2011.

49. D. Cordasco and P. Bagchi. 'Intermittency and synchronized motion in red blood cell dynamics in shear flow'. J. Fluid Mech. 759:472-488 (2014).

50. G.B. Jeffery. 'The motion of ellipsoidal particles immersed in a viscous fluid.' Proceedings Royal Soc. London 102:161-179 (1922).

51. A. Goldman, R. Cox, and H. Brenner. 'Slow viscous motion of a sphere parallel to a plane wall - II. Couette flow.' Chem. Eng. Sci. 22:653-660 (1967).

52. S. Wakiya, C. Darabaner, and S. Mason. 'Particle motions in sheared suspensions XXI: Interactions of rigid spheres (theoretical).' Rheol. Acta 6:264-273 (1967).

53. M. Chaoui and F. Feuillebois. 'Creeping flow around a sphere in a shear flow close to a wall.' Q. J. Mech. Appl. Math. 56:381-410 (2003).

54. R. Waugh and E.A. Evans. 'Thermoelasticity of red blood cell membrane.' Biophys. J. 26:115-132 (1979).

55. A.R. Pries, K. Ley, M. Claassen, and P. Gaehtgens. 'Red cell distribution at microvascular bifurcations.' Microvascular Res. 38:81-101 (1989).

56. S. Yang, A. Undar, and J. Zahn. 'A microfluidic device for continuous, real time blood plasma separation.' Lab Chip 6:871-880 (2006).

57. P.K. Ong, S. Jain, and S. Kim. 'Spatio-temporal variations in cell-free layer formation near bifurcations of small arterioles'. Microvascular Res. 83:118-125 (2012).

58. S. Nagrath et al. 'Isolation of rare circulating tumour cells in cancer patients by microchip technology'. Nature. 450:1235-1239 (2007).

59. C.T. Culbertson, T.G. Mickleburgh, S.A. Stewart-James, K.A. Sellens, and M. Pressnall. 'Micro total analysis systems: Fundamental advances and biological applications'. Anal. Chem. 86:95-118 (2014).

60. R.T. Carr, J.B. Geddes, and F. Wu. 'Oscillations in a simple microvascular network'. Annals Biomed Eng. 33:764-771 (2005).

61. J.H. Ferziger, M. Peric. 'Computational methods for fluid dynamics.' 3rd, rev.ed. Springer (2002).

62. S. Jadhav, C. Eggleton, K. Konstantopoulos. 'A 3-D computational model predicts that cell deformation affects selectin-mediated leukocyte rolling.' Biophys. J. 88:96-104 (2005).

63. J. Freund. 'Leukocyte margination in a model microvessel.' Phys. Fluids, 19:023301 (2007).

64. V. Pappu, P. Bagchi. 'Hydrodynamic interaction between erythrocytes and leukocytes affects rheology of blood in microvessels.' Biorheology, 44(3):191-215 (2007).

65. V. Pappu, P. Bagchi, 'Three-dimensional computational modeling and simulation of leukocyte rolling adhesion and deformation.' Comp. Biol. Med. 38:738-753 (2008).

66. V. Pappu, S. Doddi, P. Bagchi. 'A computational study of leukocyte adhesion and its effects on flow patterns in microvessels.' J. Theor. Biol. 254:483-498 (2008).

67. B. Leimkuhler, X. Shang. 'On the numerical treatment of dissipative particle dynamics and related systems.' J. Comput. Phys. 280:72-95 (2015).

68. R. Groot, P. Warren. 'Dissipative particle dynamics: Bridging the gap between atomistic and mesoscopic simulation.' J. Chem. Phys. 107:4423-4435 (1997).

69. C. Pozrikidis. Modeling and Simulation of Capsules and Biological Cells. Chapman \& Hall/CRC. (2003). 
70. D.B. Khismatullin, G.A. Truskey. 'Three-dimensional numerical simulation of receptor-mediated leukocyte adhesion to surfaces: effects of cell deformability and viscoelasticity'. Phys. Fluids 17:031505 (2005).

71. M.R. King, D.A. Hammer. 'Multiparticle adhesive dynamics: hydrodynamic recruitment of rolling leukocytes'. Proc. Natl Acad. Sci. USA 98:12924 (2001).

72. M.R. King, D.A. Hammer. 'Multiparticle adhesive dynamics. Interactions between stably rolling cells'. Biophys. J. 81:799-813 (2001). 


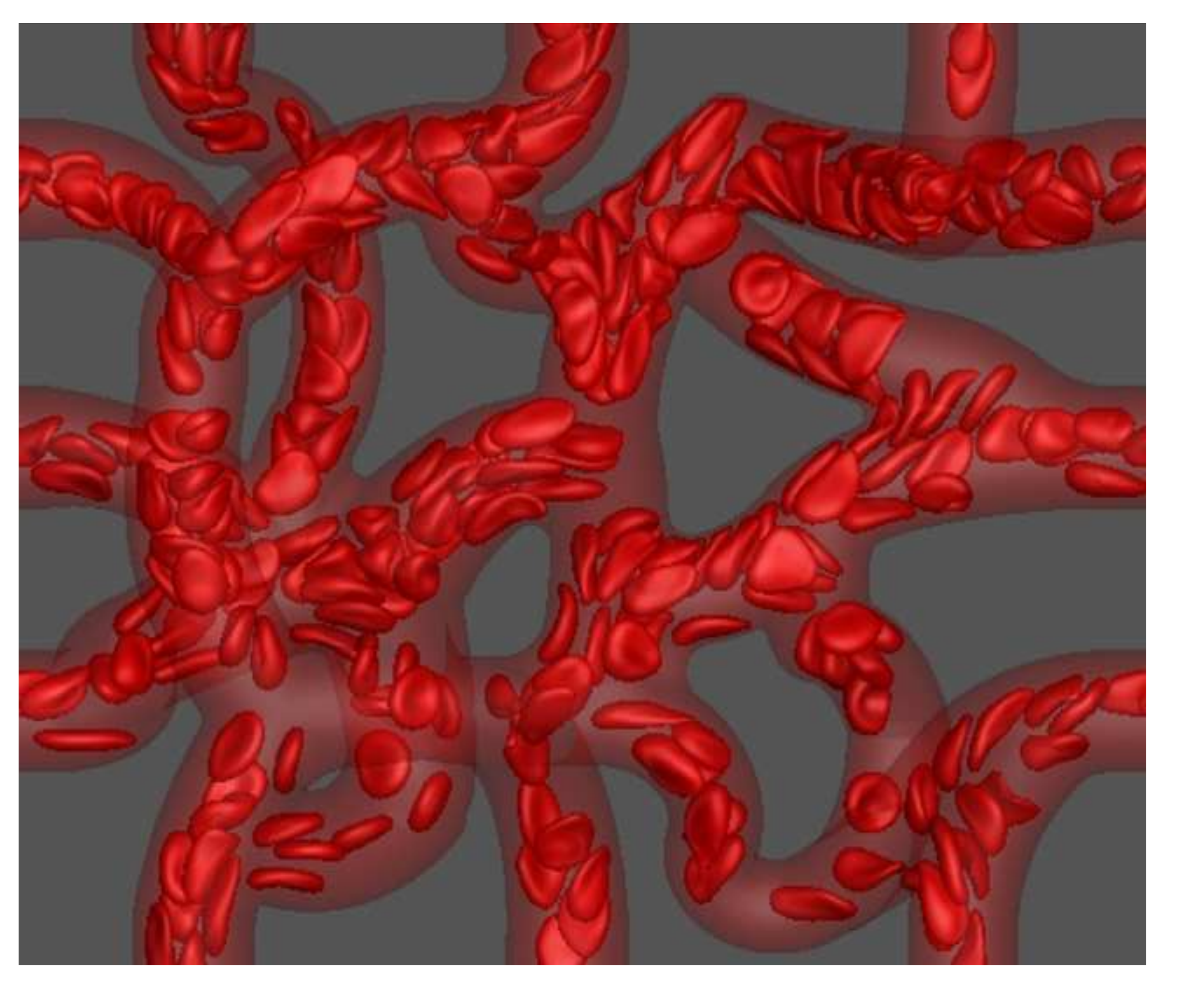

. 